\title{
Nucleon-nucleon scattering in a three dimensional approach
}

\author{
I. Fachruddin, ${ }^{1}$ Ch. Elster, ${ }^{2}$ and W. Glöckle ${ }^{1}$ \\ ${ }^{1}$ Institute for Theoretical Physics II, Ruhr-University Bochum, D-44780 Bochum, Germany \\ ${ }^{2}$ Institut für Kernphysik, Forschungszentrum Jülich, Germany D-52425 Jülich, Germany \\ and Institute of Nuclear and Particle Physics, Ohio University, Athens, Ohio 45701
}

(Received 25 April 2000; published 14 September 2000)

\begin{abstract}
The nucleon-nucleon $(N N) t$ matrix is calculated directly as function of two vector momenta for different realistic $N N$ potentials. To facilitate this a formalism is developed for solving the two-nucleon LippmannSchwinger equation in momentum space without employing a partial wave decomposition. The total spin is treated in a helicity representation. Two different realistic $N N$ interactions, one defined in momentum space and one in coordinate space, are presented in a form suited for this formulation. The angular and momentum dependence of the full amplitude is studied and displayed. A partial wave decomposition of the full amplitude it carried out to compare the presented results with the well-known phase shifts provided by those interactions.
\end{abstract}

PACS number(s): 21.45.+v, 13.75.Cs

\section{INTRODUCTION}

Experience in three- and four-nucleon calculations [1-3] shows that the standard treatment based on a partial wave projected momentum space basis is quite successful but also rather tedious, since each building block requires extended algebra. For example, the representation of the various permutation operators (i.e., the transformations among angular momenta belonging to different Jacobi momenta) is very involved [4] and requires intricate numerical realizations [5]. On the other hand, we demonstrated in Ref. [6] the relative ease with which a three-boson bound state could be calculated in the Faddeev scheme avoiding an angular momentum decomposition altogether. Instead of solving a large set of coupled two dimensional integral equations in the standard partial wave framework, in a three dimensional (3D) formulation only one single integral equation in three dimensions had to be solved. We had the same positive experience with the scattering of three bosons [7], where the algebraic formulation and numerical implementation of the one Faddeevequation was much simpler than the machinery of the approach based on partial wave decomposition.

The input to these three-body calculations without angular momentum decomposition is two-body $t$ matrices, which are off the energy shell. In addition to the off-shell energy they depend on the magnitudes of the initial and final momenta and the angle between the two momenta. In Ref. [8] we showed that the two-body $t$ matrices can be obtained very easily by solving a two dimensional Lippmann-Schwinger (LS) equation instead of preparing and handling quite a few one dimensional LS equations for each angular momentum state separately. The off-shell energies required in a threebody calculation lie between the total three-body energy and minus infinity. Interestingly we found in Ref. [8] that the $t$ matrix at large negative energies is nearly equal to the real part of the $t$ matrix at corresponding positive energy. This insight, which was also pointed out in Ref. [9], explains directly that for bound state calculations a surprisingly large number of partial waves is necessary for convergence. In addition, recent three-nucleon $(3 N)$ Faddeev calculations $[10]$ based on realistic nucleon-nucleon $(N N)$ forces and per- formed between 100 and $200-\mathrm{MeV}$ nucleon laboratory energy reach the very limits of present day computational resources, since the number of angular momentum states involved increases dramatically. Regarding all the above considerations it is a challenge to incorporate spin into the previous calculations [6-8] and perform few-nucleon calculations without angular momentum decomposition. As an aside, in the Monte Carlo calculations in configuration space [11] this is anyhow a standard procedure.

In the case of $N N$ scattering calculations without partial wave decomposition have already been realized several times $[12,13]$. In those studies the spin states were treated in their individual $m$ representations. A different approach is based on a helicity representation [14], where the helicity related to the total two-nucleon spin was introduced. In the present work we follow this basic philosophy, however, we end up with somewhat different final equations to be solved. We apply our formulation to the realistic $N N$ potentials Bonn-B [15], as well as the Argonne AV18 [16] potential and check the accuracy of our calculations by comparing to phase shift parameters obtained from corresponding partial wave calculations. The potentials we chose for implementation are given in an operator form, and thus directly applicable to our formulation without partial wave decomposition. Other modern $N N$ forces, like Nijm I and II (Ref. [17]) or CD-Bonn (Ref. [18]), are parametrized for each angular momentum state separately and thus are not as useful for an approach without partial wave decomposition.

This article is structured in the following way: In Sec. II we present the formalism. The helicity representation of the potentials and the final form of the Lippmann-Schwinger equations are displayed in Sec. III. The implementation of two modern realistic $N N$ forces into our formulation is given in Sec. IV. The connection to the standard partial wave representation is given in Sec. V. Our calculations and results are presented in Sec. VI, and we conclude in Sec. VII.

\section{FORMULATION FOR TWO NUCLEON SCATTERING BASED ON HELICITIES}

First, we introduce a helicity basis for the total spin $S$ of two nucleons. For the $z$ axis being quantization axis, the total 
spin state has the well-known form

$$
|\hat{z} s \Lambda\rangle=\sum_{m_{1} m_{2}} C\left(\frac{1}{2} \frac{1}{2} S ; m_{1} m_{2} \Lambda\right)\left|\hat{z} \frac{1}{2} m_{1}\right\rangle\left|\hat{z} \frac{1}{2} m_{2}\right\rangle .
$$

Applying the rotation operator

$$
R(\hat{q})=e^{-i S_{z} \phi} e^{-i S_{y} \theta}
$$

where $S_{z}, S_{y}$ are components of the spin operator $\mathbf{S}=\frac{1}{2}\left(\boldsymbol{\sigma}_{1}\right.$ $\left.+\boldsymbol{\sigma}_{2}\right)$, leads to the general state

$$
|\hat{q} S \Lambda\rangle=R(\hat{q})|\hat{z} S \Lambda\rangle
$$

This is eigenstate to the helicity operator $\mathbf{S} \cdot \hat{q}$ :

$$
\mathbf{S} \cdot \hat{q}|\hat{q} S \Lambda\rangle=\Lambda|\hat{q} S \Lambda\rangle
$$

This follows simply from $R(\hat{q}) \mathbf{S} \cdot \hat{z} R^{-1}(\hat{q})=\mathbf{S} \cdot \hat{q}$. Next we define momentum-helicity states as

$$
|\mathbf{q} ; \hat{q} S \Lambda\rangle \equiv|\mathbf{q}\rangle|\hat{q} S \Lambda\rangle
$$

where $\mathbf{q}$ is the relative momentum of the two nucleons.

The parity operator $P$ acts as

$$
P|\mathbf{q} ; \hat{q} S \Lambda\rangle=|-\mathbf{q} ; \hat{q} S \Lambda\rangle
$$

on the momentum helicity eigenstates. Consequently parity eigenstates are given as

$$
|\mathbf{q} ; \hat{q} S \Lambda\rangle_{\pi}=\frac{1}{\sqrt{2}}\left(1+\eta_{\pi} P\right)|\mathbf{q} ; \hat{q} S \Lambda\rangle
$$

where $\eta_{\pi}= \pm 1$.

Combining Eq. (2.7) with two-body isospin states $\left|t m_{t}\right\rangle$, we introduce antisymmetrized two-nucleon states as

$$
\begin{aligned}
|\mathbf{q} ; \hat{q} S \Lambda ; t\rangle^{\pi a} & =\frac{1}{\sqrt{2}}\left(1-P_{12}\right) \frac{1}{\sqrt{2}}\left(1+\eta_{\pi} P\right)|\mathbf{q} ; \hat{q} S \Lambda\rangle|t\rangle \\
& =\frac{1}{\sqrt{2}}\left[1-\eta_{\pi}(-)^{S+t}\right]|t\rangle|\mathbf{q} ; \hat{q} S \Lambda\rangle_{\pi} .
\end{aligned}
$$

To arrive at Eq. (2.8) we use well-known properties of twonucleon spin and isospin states.

In order to evaluate the normalization of the states in Eq. (2.8) we need the relation between $|\hat{q} S \Lambda\rangle$ and $|-\hat{q} S \Lambda\rangle$. We use the definition of Eq. (2.3) for $|-\hat{q} S \Lambda\rangle$ and the Wigner $D$ function, namely

$$
D_{\Lambda^{\prime} \Lambda}^{S}(\hat{q})=\left\langle\hat{z} S \Lambda^{\prime}|R(\hat{q})| \hat{z} S \Lambda\right\rangle=e^{-i \Lambda^{\prime} \phi} d_{\Lambda^{\prime} \Lambda}^{S}(\theta),
$$

to obtain

$$
\begin{aligned}
|-\hat{q} S \Lambda\rangle & =\sum_{\Lambda^{\prime}} D_{\Lambda^{\prime} \Lambda}^{S}(-\hat{q})\left|\hat{z} S \Lambda^{\prime}\right\rangle \\
& =\sum_{\Lambda^{\prime}} e^{-i(\phi+\pi) \Lambda^{\prime}} d_{\Lambda^{\prime} \Lambda}^{S}(\pi-\theta)\left|\hat{z} S \Lambda^{\prime}\right\rangle \\
& =\sum_{\Lambda^{\prime}} e^{-i(\phi+\pi) \Lambda^{\prime}}(-)^{S+\Lambda^{\prime}} d_{\Lambda^{\prime},-\Lambda}^{S}(\theta)\left|\hat{z} S \Lambda^{\prime}\right\rangle \\
& =(-)^{S} \sum_{\Lambda^{\prime}} D_{\Lambda^{\prime},-\Lambda}^{S}(\phi \theta 0)\left|\hat{z} S \Lambda^{\prime}\right\rangle \\
& =(-)^{S}|\hat{q} S-\Lambda\rangle .
\end{aligned}
$$

Now the normalization of the states given in Eq. (2.8) can be worked out as

$$
\begin{aligned}
\pi^{\prime} a\left\langle\mathbf{q}^{\prime} ; \hat{q}^{\prime} S^{\prime} \Lambda^{\prime} ; t^{\prime} \mid \mathbf{q} ; \hat{q} S \Lambda ; t\right\rangle^{\pi a} \\
=\frac{1}{2}\left[1-\eta_{\pi^{\prime}}(-)^{S^{\prime}+t^{\prime}}\right]\left[1-\eta_{\pi}(-)^{S+t}\right] \\
\quad \times \delta_{t^{\prime} t \pi^{\prime}}\left\langle\mathbf{q}^{\prime} ; \hat{q}^{\prime} S^{\prime} \Lambda^{\prime} \mid \mathbf{q} ; \hat{q} S \Lambda\right\rangle_{\pi} \\
=\left[1-\eta_{\pi}(-)^{S+t}\right] \delta_{t^{\prime} t} \delta_{\eta_{\pi^{\prime}} \eta_{\pi}} \delta_{S^{\prime} S}\left[\delta\left(\mathbf{q}^{\prime}-\mathbf{q}\right) \delta_{\Lambda^{\prime} \Lambda}\right. \\
\left.\quad+\eta_{\pi}(-)^{S} \delta\left(\mathbf{q}^{\prime}+\mathbf{q}\right) \delta_{\Lambda^{\prime},-\Lambda}\right] .
\end{aligned}
$$

Using this result it can also be verified that the completeness relation of the states defined in Eq. (2.8) takes the form

$$
\sum_{S \Lambda \pi t} \int d^{3} q|\mathbf{q} ; \hat{q} S \Lambda ; t\rangle^{\pi a} \frac{1}{4} \pi a\langle\mathbf{q} ; \hat{q} S \Lambda ; t|=1 .
$$

Equipped with the above given basis states one can formulate the Lippmann-Schwinger integral equation. We define as matrix elements

$$
\begin{gathered}
T_{\Lambda^{\prime}{ }_{\Lambda}}^{\pi S t}\left(\mathbf{q}^{\prime}, \mathbf{q}\right) \equiv{ }^{\pi a}\left\langle\mathbf{q}^{\prime} ; \hat{q}^{\prime} S \Lambda^{\prime} ; t|T| \mathbf{q} ; \hat{q} S \Lambda ; t\right\rangle^{\pi a} \\
V_{\Lambda^{\prime} \Lambda^{\pi}}^{\pi S t}\left(\mathbf{q}^{\prime}, \mathbf{q}\right) \equiv{ }^{\pi a}\left\langle\mathbf{q}^{\prime} ; \hat{q}^{\prime} S \Lambda^{\prime} ; t|V| \mathbf{q} ; \hat{q} S \Lambda ; t\right\rangle^{\pi a} .
\end{gathered}
$$

Then using Eqs. (2.12)-(2.14) the operator equation $T=V$ $+V G_{0} T$, which has a driving term the nucleon-nucleon $(N N)$ potential $V$ takes the form

$$
\begin{aligned}
T_{\Lambda^{\prime} \Lambda}^{\pi S t}\left(\mathbf{q}^{\prime}, \mathbf{q}\right)= & V_{\Lambda^{\prime} \Lambda}^{\pi S t}\left(\mathbf{q}^{\prime}, \mathbf{q}\right) \\
& +\frac{1}{4} \sum_{\Lambda^{\prime \prime}} \int d^{3} q^{\prime \prime} V_{\Lambda^{\prime} \Lambda^{\prime \prime}}^{\pi S t}\left(\mathbf{q}^{\prime}, \mathbf{q}^{\prime \prime}\right) G_{0}\left(q^{\prime \prime}\right) \\
& \times T_{\Lambda^{\prime \prime} \Lambda}^{\pi S t}\left(\mathbf{q}^{\prime \prime}, \mathbf{q}\right) .
\end{aligned}
$$

We now distinguish between the two cases for total spin $S=0$ and $S=1$. For $S=0$ Eq. (2.15) is one equation, similar to the one discussed in Ref. [8] for the bosonic case. For $S$ $=1$ there are three coupled equations to each $\Lambda=-1,0,1$. We use the property that $V$ conserves spin and isospin, which is valid to a high degree of accuracy. The coupled sets of equations in Eq. (2.15) can be further reduced as shown below. Using Eq. (2.8) and the parity invariance of $V$ one obtains 


$$
\begin{aligned}
V_{\Lambda^{\prime} \Lambda}^{\pi S t}\left(\mathbf{q}^{\prime}, \mathbf{q}\right)= & \frac{1}{2}\left[1-\eta_{\pi}(-)^{S+t}\right]^{2} \\
& \times\left\langle\left. t\right|_{\pi}\left\langle\mathbf{q}^{\prime} ; \hat{q}^{\prime} S \Lambda^{\prime}|V| \mathbf{q} ; \hat{q} S \Lambda\right\rangle_{\pi} \mid t\right\rangle \\
= & \sqrt{2}\left[1-\eta_{\pi}(-)^{S+t}\right] \\
& \times\left\langle t\left|\left\langle\hat{q}^{\prime} S \Lambda^{\prime}\left|\left\langle\mathbf{q}^{\prime}|V| \mathbf{q}\right\rangle_{\pi}\right| \hat{q} S \Lambda\right\rangle\right| t\right\rangle,
\end{aligned}
$$

where

$$
|\mathbf{q}\rangle_{\pi} \equiv \frac{1}{\sqrt{2}}\left(|\mathbf{q}\rangle+\eta_{\pi}|-\mathbf{q}\rangle\right)
$$

This expression can be connected to $V_{-\Lambda^{\prime} \Lambda}^{\pi S t}\left(\mathbf{q}^{\prime}, \mathbf{q}\right)$ using Eqs. (2.10) and (2.17) with the result

$$
V_{-\Lambda^{\prime} \Lambda}^{\pi S t}\left(\mathbf{q}^{\prime}, \mathbf{q}\right)=\eta_{\pi}(-)^{S} V_{\Lambda^{\prime} \Lambda}^{\pi S t}\left(-\mathbf{q}^{\prime}, \mathbf{q}\right)
$$

A corresponding relation is also valid for the matrix element $T_{\Lambda^{\prime} \Lambda}^{\pi S t}\left(\mathbf{q}^{\prime}, \mathbf{q}\right)$. Similarly one finds

$$
V_{\Lambda^{\prime},-\Lambda}^{\pi S t}\left(\mathbf{q}^{\prime}, \mathbf{q}\right)=\eta_{\pi}(-)^{S} V_{\Lambda^{\prime} \Lambda^{\prime}}^{\pi S t}\left(\mathbf{q}^{\prime},-\mathbf{q}\right) .
$$

We can now simplify the set of coupled equations (2.15) for $S=1$ in the following way:

$$
\begin{aligned}
& \int d^{3} q^{\prime \prime} V_{\Lambda^{\prime},-1}^{\pi S t}\left(\mathbf{q}^{\prime}, \mathbf{q}^{\prime \prime}\right) G_{0}\left(q^{\prime \prime}\right) T_{-1, \Lambda}^{\pi S t}\left(\mathbf{q}^{\prime \prime}, \mathbf{q}\right) \\
& =\int d^{3} q^{\prime \prime} \eta_{\pi}(-)^{S} V_{\Lambda^{\prime}, 1}^{\pi S t}\left(\mathbf{q}^{\prime},-\mathbf{q}^{\prime \prime}\right) \\
& \quad \times G_{0}\left(q^{\prime \prime}\right) \eta_{\pi}(-)^{S} T_{1, \Lambda}^{\pi S t}\left(-\mathbf{q}^{\prime \prime}, \mathbf{q}\right) \\
& =\int d^{3} q^{\prime \prime} V_{\Lambda^{\prime}, 1}^{\pi S t}\left(\mathbf{q}^{\prime},-\mathbf{q}^{\prime \prime}\right) G_{0}\left(q^{\prime \prime}\right) T_{1, \Lambda}^{\pi S t}\left(-\mathbf{q}^{\prime \prime}, \mathbf{q}\right) \\
& =\int d^{3} q^{\prime \prime} V_{\Lambda^{\prime}, 1}^{\pi S t}\left(\mathbf{q}^{\prime}, \mathbf{q}^{\prime \prime}\right) G_{0}\left(q^{\prime \prime}\right) T_{1, \Lambda}^{\pi S t}\left(\mathbf{q}^{\prime \prime}, \mathbf{q}\right) .
\end{aligned}
$$

This leads to

$$
\begin{aligned}
T_{\Lambda^{\prime} \Lambda}^{\pi S t}\left(\mathbf{q}^{\prime}, \mathbf{q}\right)= & V_{\Lambda^{\prime} \Lambda}^{\pi S t}\left(\mathbf{q}^{\prime}, \mathbf{q}\right) \\
& +\frac{1}{2} \int d^{3} q^{\prime \prime} V_{\Lambda^{\prime}{ }_{1}}^{\pi S t}\left(\mathbf{q}^{\prime}, \mathbf{q}^{\prime \prime}\right) G_{0}\left(q^{\prime \prime}\right) T_{1 \Lambda}^{\pi S t}\left(\mathbf{q}^{\prime \prime}, \mathbf{q}\right) \\
& +\frac{1}{4} \int d^{3} q^{\prime \prime} V_{\Lambda^{\prime} 0}^{\pi S t}\left(\mathbf{q}^{\prime}, \mathbf{q}^{\prime \prime}\right) G_{0}\left(q^{\prime \prime}\right) T_{0 \Lambda}^{\pi S t}\left(\mathbf{q}^{\prime \prime}, \mathbf{q}\right)
\end{aligned}
$$

We now have for the case $S=1$ two coupled equations, namely for $\Lambda^{\prime}=1,0$ for each $\Lambda$. Moreover due to Eq. (2.19), which is also valid for the $t$ matrix $T$, it is sufficient to consider $\Lambda=0$ and 1 . As an aside it should be mentioned that because of the relation $\pi(-)^{S+t}=-1$ the isospin quantum number $t$ is fixed, once $\pi$ and $S$ are chosen.
Finally we need to calculate the physical $T$-matrix element expressed in terms of the states

$$
\left|\nu_{1} \nu_{2} m_{1} m_{2} \mathbf{q}\right\rangle_{a} \equiv \frac{1}{\sqrt{2}}\left(1-P_{12}\right)\left|\nu_{1} \nu_{2} m_{1} m_{2} \mathbf{q}\right\rangle
$$

where $\nu_{1}, \nu_{2}$ and $m_{1}, m_{2}$ are the magnetic isospin and spin quantum numbers, $\mathbf{q}$ describes the relative momentum of the two nucleons, and $P_{12}$ is the permutation operator between the particles 1 and 2 . The physical $T$-matrix element is then given as

$$
\begin{aligned}
& { }_{a}\left\langle\nu_{1} \nu_{2} m_{1}^{\prime} m_{2}^{\prime} \mathbf{q}^{\prime}|T| \nu_{1} \nu_{2} m_{1} m_{2} \mathbf{q}\right\rangle_{a} \\
& \quad=\left\langle\nu_{1} \nu_{2} m_{1}^{\prime} m_{2}^{\prime} \mathbf{q}^{\prime}\left|T\left(1-P_{12}\right)\right| \nu_{1} \nu_{2} m_{1} m_{2} \mathbf{q}\right\rangle .
\end{aligned}
$$

The above equation now has to be expressed in terms of $T_{\Lambda^{\prime} \Lambda}^{\pi S t}\left(\mathbf{q}^{\prime}, \mathbf{q}\right)$. In doing so we encounter the matrix element

$$
\begin{aligned}
\left\langle\hat{q}^{\prime} S \Lambda^{\prime} \mid m_{1} m_{2}\right\rangle & =\sum_{S^{\prime}} C\left(\frac{1}{2} \frac{1}{2} S^{\prime} ; m_{1} m_{2} \Lambda_{0}\right)\left\langle\hat{q}^{\prime} S \Lambda^{\prime} \mid \hat{z} S^{\prime} \Lambda_{0}\right\rangle \\
& =C\left(\frac{1}{2} \frac{1}{2} S ; m_{1} m_{2} \Lambda_{0}\right) e^{i \Lambda_{0} \phi^{\prime}} d_{\Lambda^{\prime} \Lambda_{0}}^{S}\left(-\theta^{\prime}\right) \\
& =C\left(\frac{1}{2} \frac{1}{2} S ; m_{1} m_{2} \Lambda_{0}\right) e^{i \Lambda_{0} \phi^{\prime}} d_{\Lambda_{0} \Lambda^{\prime}}\left(\theta^{\prime}\right) .
\end{aligned}
$$

After some straightforward algebra one finds

$$
\begin{aligned}
{ }^{\pi a}\left\langle\mathbf{q}^{\prime} ;\right. & \hat{q}^{\prime} S \Lambda^{\prime} ; t\left|\nu_{1} \nu_{2} m_{1} m_{2} \mathbf{q}\right\rangle_{a} \\
= & \frac{1}{\sqrt{2}}\left[1-\eta_{\pi}(-)^{S+t}\right] \frac{1}{\sqrt{2}}\left\langle t \mid \nu_{1} \nu_{2}\right\rangle \\
& \times{ }_{\pi}\left\langle\mathbf{q}^{\prime} ; \hat{q}^{\prime} S \Lambda^{\prime}\left|\left(1-P_{12}\right)\right| m_{1} m_{2} \mathbf{q}\right\rangle \\
= & \frac{1}{\sqrt{2}} C\left(\frac{1}{2} \frac{1}{2} t ; \nu_{1} \nu_{2}\right) C\left(\frac{1}{2} \frac{1}{2} S ; m_{1} m_{2} \Lambda_{0}\right) \\
& \times e^{i \Lambda_{0} \phi^{\prime}} d_{\Lambda_{0} \Lambda^{\prime}}\left(\theta^{\prime}\right)\left[1-\eta_{\pi}(-)^{S+t}\right] \\
& \times\left[\delta\left(\mathbf{q}^{\prime}-\mathbf{q}\right)+\eta_{\pi} \delta\left(\mathbf{q}^{\prime}+\mathbf{q}\right)\right] .
\end{aligned}
$$

We then insert the completeness relation, Eq. (2.12), twice into Eq. (2.23), and using Eq. (2.25) obtain after some algebra

$$
\begin{aligned}
{ }_{a}\left\langle\nu_{1}\right. & \left.\nu_{2} m_{1}^{\prime} m_{2}^{\prime} \mathbf{q}^{\prime}|T| \nu_{1} \nu_{2} m_{1} m_{2} \mathbf{q}\right\rangle_{a} \\
= & \frac{1}{4} e^{-i\left(\Lambda_{0}^{\prime} \phi^{\prime}-\Lambda_{0} \phi\right)} \sum_{S \pi t}\left[1-\eta_{\pi}(-)^{S+t}\right] C\left(\frac{1}{2} \frac{1}{2} t ; \nu_{1} \nu_{2}\right)^{2} \\
& \times C\left(\frac{1}{2} \frac{1}{2} S ; m_{1}^{\prime} m_{2}^{\prime} \Lambda_{0}^{\prime}\right) C\left(\frac{1}{2} \frac{1}{2} S ; m_{1} m_{2} \Lambda_{0}\right) \\
& \times \sum_{\Lambda^{\prime} \Lambda} d_{\Lambda_{0}^{\prime} \Lambda^{\prime}}\left(\theta^{\prime}\right) d_{\Lambda_{0} \Lambda}^{S}(\theta) T_{\Lambda^{\prime} \Lambda}^{\pi S t}\left(\mathbf{q}^{\prime}, \mathbf{q}\right) .
\end{aligned}
$$


For our calculation we choose $\hat{q}=\hat{z}$ and use $d_{\Lambda_{0} \Lambda}^{S}(0)$ $=\delta_{\Lambda_{0} \Lambda}$. Furthermore, as will be shown in Sec. III, the azimuthal dependence of $\mathbf{q}^{\prime}$ and $\mathbf{q}$ can be factored out and one finds

$$
T_{\Lambda^{\prime} \Lambda}^{\pi S t}\left(\mathbf{q}^{\prime}, \mathbf{q}\right)=e^{i \Lambda\left(\phi^{\prime}-\phi\right)} T_{\Lambda^{\prime} \Lambda^{\prime}}^{\pi S t}\left(q^{\prime}, q, \theta^{\prime}\right)
$$

With this Eq. (2.26) can be written as (note $q^{\prime}=q$ )

$$
\begin{aligned}
& { }_{a}\left\langle\nu_{1} \nu_{2} m_{1}^{\prime} m_{2}^{\prime} q \hat{q}^{\prime}|T| \nu_{1} \nu_{2} m_{1} m_{2} \mathbf{q}\right\rangle_{a} \\
& =\frac{1}{4} e^{-i\left(\Lambda_{0}^{\prime}-\Lambda_{0}\right) \phi^{\prime}} \sum_{S \pi t} C\left(\frac{1}{2} \frac{1}{2} t ; \nu_{1} \nu_{2}\right)^{2} \\
& \quad \times\left[1-\eta_{\pi}(-)^{S+t}\right] C\left(\frac{1}{2} \frac{1}{2} S ; m_{1}^{\prime} m_{2}^{\prime} \Lambda_{0}^{\prime}\right) \\
& \quad \times C\left(\frac{1}{2} \frac{1}{2} S ; m_{1} m_{2} \Lambda_{0}\right) \sum_{\Lambda^{\prime}} d_{\Lambda_{0}^{\prime} \Lambda^{\prime}}^{S}\left(\theta^{\prime}\right) T_{\Lambda^{\prime} \Lambda_{0}}^{\pi S t}\left(q, q, \theta^{\prime}\right) .
\end{aligned}
$$

\section{GENERAL STRUCTURE OF THE POTENTIAL OPERATOR AND FINAL FORM OF THE SCATTERING EQUATION}

As is well known, rotational-, parity-, and time reversal invariance restricts any $N N$ potential $V$ to be formed out of six terms [20], which are given by

$$
\begin{gathered}
W_{1}=1, \\
W_{2}=\left(\boldsymbol{\sigma}_{1}+\boldsymbol{\sigma}_{2}\right) \cdot \hat{N} \\
W_{3}=\boldsymbol{\sigma}_{1} \cdot \hat{N} \boldsymbol{\sigma}_{2} \cdot \hat{N} \\
W_{4}=\boldsymbol{\sigma}_{1} \cdot \hat{P} \boldsymbol{\sigma}_{2} \cdot \hat{P} \\
W_{5}=\boldsymbol{\sigma}_{1} \cdot \hat{K} \boldsymbol{\sigma}_{2} \cdot \hat{K} \\
W_{6}=\boldsymbol{\sigma}_{1} \cdot \hat{P} \boldsymbol{\sigma}_{2} \cdot \hat{K}+\boldsymbol{\sigma}_{1} \cdot \hat{K} \boldsymbol{\sigma}_{2} \cdot \hat{P}
\end{gathered}
$$

where the unit vectors $\hat{N}, \hat{P}$, and $\hat{K}$ are defined in terms of the relative momenta $\mathbf{q}$ and $\mathbf{q}^{\prime}$ as

$$
\begin{gathered}
\hat{N}=\frac{\left(\mathbf{q} \times \mathbf{q}^{\prime}\right)}{\left|\mathbf{q} \times \mathbf{q}^{\prime}\right|} \\
\hat{P}=\frac{\left(\mathbf{q}^{\prime}+\mathbf{q}\right)}{\left|\mathbf{q}^{\prime}+\mathbf{q}\right|} \\
\hat{K}=\frac{\left(\mathbf{q}^{\prime}-\mathbf{q}\right)}{\left|\mathbf{q}^{\prime}-\mathbf{q}\right|} .
\end{gathered}
$$

The most general potential is then given as linear combination of those operators, namely

$$
\left\langle\mathbf{q}^{\prime}|V| \mathbf{q}\right\rangle \equiv V\left(\mathbf{q}^{\prime}, \mathbf{q}\right)=\sum_{i=1}^{6} v_{i}\left(q^{\prime}, q, \gamma\right) W_{i}
$$

where $v_{i}\left(q^{\prime}, q, \gamma\right)$ are scalar functions depending on the magnitudes of the vectors $\mathbf{q}^{\prime}, \mathbf{q}$, and the angle between the two, $\gamma \equiv \hat{q}^{\prime} \cdot \hat{q}$. In Sec. IV we will provide specific realizations of the potential $V\left(\mathbf{q}^{\prime}, \mathbf{q}\right)$.

The evaluation of the matrix elements of Eq. (2.14) for the set of operators $W_{i}$ is in principle possible. However, it is simpler to define a different set of six independent operators, $\Omega_{i}$, which is more adapt to our choice of basis states. We introduce the following set of operators:

$$
\begin{gathered}
\Omega_{1}=1, \\
\Omega_{2}=\mathbf{S}^{2}, \\
\Omega_{3}=\mathbf{S} \cdot \hat{q}^{\prime} \mathbf{S} \cdot \hat{q}^{\prime}, \\
\Omega_{4}=\mathbf{S} \cdot \hat{q}^{\prime} \mathbf{S} \cdot \hat{q}, \\
\Omega_{5}=\left(\mathbf{S} \cdot \hat{q}^{\prime}\right)^{2}(\mathbf{S} \cdot \hat{q})^{2}, \\
\Omega_{6}=\mathbf{S} \cdot \hat{q} \mathbf{S} \cdot \hat{q} .
\end{gathered}
$$

The two sets of operators given in Eqs. (3.1) and (3.6) can be easily related via

$$
W_{i}=\sum_{j} A_{i j} \Omega_{j}
$$

The transformation matrix $A_{i j}$ and its inverse are explicitly given in Appendix A.

Using the operators $\Omega_{i}$, the spin-dependent part of the matrix elements of Eq. (2.14) can then be reduced to the evaluation of matrix elements of the type $\left\langle\hat{q}^{\prime} S \Lambda^{\prime}\left|\Omega_{i}\right| \hat{q} S \Lambda\right\rangle$, namely

$$
\begin{gathered}
\left\langle\hat{q}^{\prime} S \Lambda^{\prime}\left|\Omega_{1}\right| \hat{q} S \Lambda\right\rangle=\left\langle\hat{q}^{\prime} S \Lambda^{\prime} \mid \hat{q} S \Lambda\right\rangle, \\
\left\langle\hat{q}^{\prime} S \Lambda^{\prime}\left|\Omega_{2}\right| \hat{q} S \Lambda\right\rangle=S(S+1)\left\langle\hat{q}^{\prime} S \Lambda^{\prime} \mid \hat{q} S \Lambda\right\rangle, \\
\left\langle\hat{q}^{\prime} S \Lambda^{\prime}\left|\Omega_{3}\right| \hat{q} S \Lambda\right\rangle=\Lambda^{\prime 2}\left\langle\hat{q}^{\prime} S \Lambda^{\prime} \mid \hat{q} S \Lambda\right\rangle, \\
\left\langle\hat{q}^{\prime} S \Lambda^{\prime}\left|\Omega_{4}\right| \hat{q} S \Lambda\right\rangle=\Lambda^{\prime} \Lambda\left\langle\hat{q}^{\prime} S \Lambda^{\prime} \mid \hat{q} S \Lambda\right\rangle, \\
\left\langle\hat{q}^{\prime} S \Lambda^{\prime}\left|\Omega_{5}\right| \hat{q} S \Lambda\right\rangle=\Lambda^{\prime 2} \Lambda^{2}\left\langle\hat{q}^{\prime} S \Lambda^{\prime} \mid \hat{q} S \Lambda\right\rangle, \\
\left\langle\hat{q}^{\prime} S \Lambda^{\prime}\left|\Omega_{6}\right| \hat{q} S \Lambda\right\rangle=\Lambda^{2}\left\langle\hat{q}^{\prime} S \Lambda^{\prime} \mid \hat{q} S \Lambda\right\rangle .
\end{gathered}
$$

Now we are left with determining the overlap of the states 
defined in Eq. (2.3). Using Eq. (2.9) this gives

$$
\begin{aligned}
\left\langle\hat{q}^{\prime} S \Lambda^{\prime} \mid \hat{q} S \Lambda\right\rangle & =\sum_{M}\left\langle\hat{q}^{\prime} S \Lambda^{\prime} \mid \hat{z} S M\right\rangle\langle\hat{z} S M \mid \hat{q} S \Lambda\rangle \\
& =\sum_{M} D_{M \Lambda^{\prime}}^{S^{*}}\left(\phi^{\prime} \theta^{\prime} 0\right) D_{M \Lambda}^{S}(\phi \theta 0) \\
& =\sum_{M=-S}^{S} e^{i M\left(\phi^{\prime}-\phi\right)} d_{M \Lambda^{\prime}}^{S}\left(\theta^{\prime}\right) d_{M \Lambda}^{S}(\theta) .
\end{aligned}
$$

A special case is the situation where $\mathbf{q}$ points in the $z$ direction, where one obtains the simple form

$$
\left\langle\hat{q}^{\prime} S \Lambda^{\prime} \mid \hat{z} S \Lambda\right\rangle=e^{i \Lambda\left(\phi^{\prime}-\phi\right)} d_{\Lambda \Lambda^{\prime}}^{S}\left(\theta^{\prime}\right) .
$$

With these preliminaries the potential matrix element of Eq. (2.14) can be written as

$$
\begin{aligned}
V_{\Lambda^{\prime} \Lambda}^{\pi S t}\left(\mathbf{q}^{\prime}, \mathbf{q}\right)= & \sqrt{2}\left[1-\eta_{\pi}(-)^{S+t}\right] \sum_{i j} A_{i j}\left\langle\hat{q}^{\prime} S \Lambda^{\prime}\left|\Omega_{j}\right| \hat{q} S \Lambda\right\rangle \\
& \times\left\langle t\left|\left\langle\mathbf{q}^{\prime}\left|v_{i}\right| \mathbf{q}\right\rangle_{\pi}\right| t\right\rangle .
\end{aligned}
$$

The coefficients $A_{i j}$ and the matrix elements $\left\langle\mathbf{q}^{\prime}\left|v_{i}\right| \mathbf{q}\right\rangle_{\pi}$ depend on the angle between $\mathbf{q}$ and $\mathbf{q}^{\prime}$ and thus on $\gamma$. Therefore their azimuthal dependence is determined by $\cos \left(\phi^{\prime}-\phi\right)$. Furthermore, the matrix elements $\Omega_{j}$ depend on the angles $\phi^{\prime}$ and $\phi$ as shown in Eq. (3.9). Thus we can schematically indicate the azimuthal dependence of the potential matrix elements as

$$
V_{\Lambda^{\prime} \Lambda}^{\pi S t}\left(\mathbf{q}^{\prime}, \mathbf{q}\right) \equiv V_{\Lambda^{\prime} \Lambda}^{\pi S t}\left\{e^{i M\left(\phi^{\prime}-\phi\right)}, \cos \left(\phi^{\prime}-\phi\right)\right\} .
$$

For the special case $\hat{q}=\hat{z}$ this reduces to the simpler form

$$
V_{\Lambda^{\prime} \Lambda}^{\pi S t}\left(\mathbf{q}^{\prime}, \mathbf{q}\right)=e^{i \Lambda\left(\phi^{\prime}-\phi\right)} V_{\Lambda^{\prime} \Lambda}^{\pi S t}\left(q^{\prime}, q, \theta^{\prime}\right),
$$

which is the driving term in the coupled set of Eqs. (2.21).

We assume that this simple dependence on $\phi^{\prime}$ and $\phi$ given in Eq. (3.13) carries over to the solution of the integral equation and choose as ansatz

$$
T_{\Lambda^{\prime} \Lambda}^{\pi S t}\left(\mathbf{q}^{\prime}, \mathbf{q}\right)=e^{i \Lambda\left(\phi^{\prime}-\phi\right)} T_{\Lambda^{\prime} \Lambda}^{\pi S t}\left(q^{\prime}, q, \theta^{\prime}\right)
$$

Inserting this into Eq. (2.15) one obtains

$$
\begin{aligned}
T_{\Lambda^{\prime} \Lambda}^{\pi S t}\left(\mathbf{q}^{\prime}, q \hat{z}\right)= & e^{i \Lambda\left(\phi^{\prime}-\phi\right)} V_{\Lambda^{\prime} \Lambda}^{\pi S t}\left(q^{\prime}, q, \theta^{\prime}\right) \\
& +\frac{1}{4} \sum_{\Lambda^{\prime \prime}} \int d^{3} q^{\prime \prime} V_{\Lambda^{\prime} \Lambda}^{\pi S t}\left\{e^{i M\left(\phi^{\prime}-\phi^{\prime \prime}\right)}, \cos \left(\phi^{\prime}-\phi^{\prime \prime}\right), q^{\prime}, q^{\prime \prime}\right\} G_{0}\left(q^{\prime \prime}\right) e^{i \Lambda\left(\phi^{\prime \prime}-\phi\right)} T_{\Lambda^{\prime \prime} \Lambda}^{\pi S t}\left(q^{\prime \prime}, q, \theta^{\prime \prime}\right) \\
= & e^{i \Lambda\left(\phi^{\prime}-\phi\right)} V_{\Lambda^{\prime} \Lambda}^{\pi S t}\left(q^{\prime}, q, \theta^{\prime}\right)+\frac{1}{4} e^{i \Lambda\left(\phi^{\prime}-\phi\right)} \sum_{\Lambda^{\prime \prime}} \int_{0}^{\infty} d q^{\prime \prime} q^{\prime \prime 2} \int_{0}^{\pi} d \theta^{\prime \prime} \sin \theta^{\prime \prime} \int_{0}^{2 \pi} d \phi^{\prime \prime} V_{\Lambda^{\prime} \Lambda}^{\pi S t}\left\{e^{i M\left(\phi^{\prime}-\phi^{\prime \prime}\right)},\right. \\
& \left.\times \cos \left(\phi^{\prime}-\phi^{\prime \prime}\right), q^{\prime}, q^{\prime \prime}\right\} G_{0}\left(q^{\prime \prime}\right) e^{i \Lambda\left(\phi^{\prime \prime}-\phi^{\prime}\right)} T_{\Lambda^{\prime \prime} \Lambda}^{\pi S t}\left(q^{\prime \prime}, q, \theta^{\prime \prime}\right) .
\end{aligned}
$$

The integrand is periodical with respect to $\phi^{\prime \prime}$, with the period being $2 \pi$. Consequently one can set $\phi^{\prime}=0$ for the $\phi^{\prime \prime}$ integration. This leads to

$$
\begin{aligned}
T_{\Lambda^{\prime} \Lambda}^{\pi S t}\left(\mathbf{q}^{\prime}, q \hat{z}\right)= & e^{i \Lambda\left(\phi^{\prime}-\phi\right)}\left[V_{\Lambda^{\prime} \Lambda}^{\pi S t}\left(q^{\prime}, q, \theta^{\prime}\right)\right. \\
& \left.+\frac{1}{4} \sum_{\Lambda^{\prime \prime}} \int_{0}^{\infty} d q^{\prime \prime} q^{\prime \prime 2} \int_{0}^{\pi} d \theta^{\prime \prime} \sin \theta^{\prime \prime} \int_{0}^{2 \pi} d \phi^{\prime \prime} V_{\Lambda^{\prime} \Lambda}^{\pi S t}\left\{e^{-i M \phi^{\prime \prime}}, \cos \phi^{\prime \prime}, q^{\prime}, q^{\prime \prime}\right\} G_{0}\left(q^{\prime \prime}\right) e^{i \Lambda \phi^{\prime \prime}} T_{\Lambda^{\prime \prime} \Lambda}^{\pi S t}\left(q^{\prime \prime}, q, \theta^{\prime \prime}\right)\right] \\
\equiv & e^{i \Lambda\left(\phi^{\prime}-\phi\right)} T_{\Lambda^{\prime} \Lambda}^{\pi S t}\left(q^{\prime}, q, \theta^{\prime}\right)
\end{aligned}
$$

verifying the correctness of the ansatz of Eq. (3.14).

Inserting this result into Eq. (2.21) gives

$$
\begin{aligned}
T_{\Lambda^{\prime} \Lambda}^{\pi S t}\left(q^{\prime}, q, \theta^{\prime}\right)= & V_{\Lambda^{\prime} \Lambda}^{\pi S t}\left(q^{\prime}, q, \theta^{\prime}\right)+\frac{1}{2} \int_{0}^{\infty} d q^{\prime \prime} q^{\prime \prime 2} \int_{-1}^{1} d\left(\cos \theta^{\prime \prime}\right) v_{\Lambda^{\prime} 1}^{\pi S t, \Lambda}\left(q^{\prime}, q^{\prime \prime}, \theta^{\prime}, \theta^{\prime \prime}\right) G_{0}\left(q^{\prime \prime}\right) T_{1 \Lambda}^{\pi S t}\left(q^{\prime \prime}, q, \theta^{\prime \prime}\right) \\
& +\frac{1}{4} \int_{0}^{\infty} d q^{\prime \prime} q^{\prime \prime 2} \int_{-1}^{1} d\left(\cos \theta^{\prime \prime}\right) v_{\Lambda^{\prime} 0}^{\pi S t, \Lambda}\left(q^{\prime}, q^{\prime \prime}, \theta^{\prime}, \theta^{\prime \prime}\right) G_{0}\left(q^{\prime \prime}\right) T_{0 \Lambda}^{\pi S t}\left(q^{\prime \prime}, q, \theta^{\prime \prime}\right),
\end{aligned}
$$


with

$$
v_{\Lambda^{\prime} \Lambda^{\prime \prime}}^{\pi S t, \Lambda}\left(q^{\prime}, q^{\prime \prime}, \theta^{\prime}, \theta^{\prime \prime}\right) \equiv \int_{0}^{2 \pi} d \phi^{\prime \prime} e^{-i \Lambda\left(\phi^{\prime}-\phi^{\prime \prime}\right)} V_{\Lambda^{\prime} \Lambda^{\prime \prime}}^{\pi S t}\left(\mathbf{q}^{\prime}, \mathbf{q}^{\prime \prime}\right)
$$

The driving term of Eq. (3.17) is a special case of Eq. (3.18) for $\theta^{\prime \prime}=0$ and $\Lambda^{\prime \prime}=\Lambda$ [up to a factor $(2 \pi)^{-1}$ ]. In summary, for the case $S=1$ we end up with two coupled integral equations in two variables for given $\Lambda$ values (only $\Lambda=1,0$ are necessary). The azimuthal integration over $\phi^{\prime \prime}$ can be performed independently, and does not enter the integral kernel.

The case $S=0$ is much simpler, one has only one integral equation in two variables, similar in its structure to the twoboson case discussed in Ref. [8].

\section{REPRESENTATION OF NN POTENTIALS}

In this section we want to demonstrate the relative ease with which modern $N N$ potentials that are given in operator form can be incorporated in a three dimensional formalism. Our choices of $N N$ potentials are a Bonn one-bosonexchange (OBE) potential [19] in the parametrization of
Bonn-B (Ref. [15]) and the Argonne coordinate space potential AV18 (Ref. [16]).

The OBE potential consists of pseudoscalar-, scalar-, and vector meson exchanges, derived from the corresponding Feynman diagrams. A three dimensional reduction of the Bethe-Salpeter equation is as achieved via the Blankenbecler-Sugar reduction. Details can be found in Refs. $[15,19]$. The resulting potential operators are given as

$$
\begin{aligned}
V^{s}\left(\mathbf{q}^{\prime}, \mathbf{q}\right)= & -\frac{g_{s}^{2}}{(2 \pi)^{3}} \sqrt{\frac{m}{E^{\prime}}} \sqrt{\frac{m}{E}} \bar{u}\left(\mathbf{q}^{\prime}\right) u(\mathbf{q}) \bar{u}\left(-\mathbf{q}^{\prime}\right) \\
& \times u(-\mathbf{q}) \frac{F_{s}\left[\left(\mathbf{q}^{\prime}-\mathbf{q}\right)^{2}\right]}{\left(\mathbf{q}^{\prime}-\mathbf{q}\right)^{2}+\mu_{s}^{2}} \\
V^{p s}\left(\mathbf{q}^{\prime}, \mathbf{q}\right)= & \frac{g_{p s}^{2}}{(2 \pi)^{3}} \sqrt{\frac{m}{E^{\prime}}} \sqrt{\frac{m}{E}} \bar{u}\left(\mathbf{q}^{\prime}\right) \gamma^{5} u(\mathbf{q}) \bar{u}\left(-\mathbf{q}^{\prime}\right) \gamma^{5} \\
& \times u(-\mathbf{q}) \frac{F_{p s}\left[\left(\mathbf{q}^{\prime}-\mathbf{q}\right)^{2}\right]}{\left(\mathbf{q}^{\prime}-\mathbf{q}\right)^{2}+\mu_{p s}^{2}}
\end{aligned}
$$

$$
\begin{aligned}
V^{v}\left(\mathbf{q}^{\prime}, \mathbf{q}\right)= & \frac{F_{v}\left[\left(\mathbf{q}^{\prime}-\mathbf{q}\right)^{2}\right]}{\left(\mathbf{q}^{\prime}-\mathbf{q}\right)^{2}+\mu_{v}^{2}} \sqrt{\frac{m}{E^{\prime}}} \sqrt{\frac{m}{E}} \frac{1}{(2 \pi)^{3}}\left(g_{v}^{2} \bar{u}\left(\mathbf{q}^{\prime}\right) \gamma^{\mu} u(\mathbf{q}) \bar{u}\left(-\mathbf{q}^{\prime}\right) \gamma_{\mu} u(-\mathbf{q})+\frac{f_{v}^{2}}{4 m^{2}}\left\{4 m^{2} \bar{u}\left(\mathbf{q}^{\prime}\right) \gamma^{\mu} u(\mathbf{q}) \bar{u}\left(-\mathbf{q}^{\prime}\right) \gamma_{\mu}\right.\right. \\
& \times u(-\mathbf{q})-2 m \bar{u}\left(\mathbf{q}^{\prime}\right) \gamma^{\mu} u(\mathbf{q}) \bar{u}\left(-\mathbf{q}^{\prime}\right)\left[\left(E^{\prime}-E\right)\left(g_{\mu}^{0}-\gamma_{\mu} \gamma^{0}\right)+\left(p_{2}+p_{2}^{\prime}\right) \mu\right] u(-\mathbf{q})-2 m \bar{u}\left(\mathbf{q}^{\prime}\right) \\
& \times\left[\left(E^{\prime}-E\right)\left(g^{0 \mu}-\gamma^{\mu} \gamma^{0}\right)+\left(p_{1}+p_{1}^{\prime}\right)^{\mu}\right] u(\mathbf{q}) \bar{u}\left(-\mathbf{q}^{\prime}\right) \gamma_{\mu} u(-\mathbf{q})+\bar{u}\left(\mathbf{q}^{\prime}\right)\left[\left(E^{\prime}-E\right)\left(g^{0 \mu}-\gamma^{\mu} \gamma^{0}\right)\right. \\
& \left.\left.+\left(p_{1}+p_{1}^{\prime}\right)^{\mu}\right] u(\mathbf{q}) \bar{u}\left(-\mathbf{q}^{\prime}\right)\left[\left(E^{\prime}-E\right)\left(g_{\mu}^{0}-\gamma_{\mu} \gamma^{0}\right)+\left(p_{2}+p_{2}^{\prime}\right)_{\mu}\right] u(-\mathbf{q})\right\}+\frac{g_{v} f_{v}}{2 m}\left\{4 m \bar{u}\left(\mathbf{q}^{\prime}\right) \gamma^{\mu} u(\mathbf{q}) \bar{u}\left(-\mathbf{q}^{\prime}\right) \gamma_{\mu}\right. \\
& \times u(-\mathbf{q})-\bar{u}\left(\mathbf{q}^{\prime}\right) \gamma^{\mu} u(\mathbf{q}) \bar{u}\left(-\mathbf{q}^{\prime}\right)\left[\left(E^{\prime}-E\right)\left(g_{\mu}^{0}-\gamma_{\mu} \gamma^{0}\right)+\left(p_{2}+p_{2}^{\prime}\right){ }_{\mu}\right] u(-\mathbf{q})-\bar{u}\left(\mathbf{q}^{\prime}\right)\left[\left(E^{\prime}-E\right)\left(g^{0 \mu}-\gamma^{\mu} \gamma^{0}\right)\right. \\
& \left.\left.\left.+\left(p_{1}+p_{1}^{\prime}\right)^{\mu}\right] u(\mathbf{q}) \bar{u}\left(-\mathbf{q}^{\prime}\right) \gamma_{\mu} u(-\mathbf{q})\right\}\right)
\end{aligned}
$$

Here $m$ stands for the nucleon mass. In the case of the vector potential one has $\left(p_{1}+p_{1}^{\prime}\right)^{\mu}=\left(E+E^{\prime}, \mathbf{q}+\mathbf{q}^{\prime}\right)$ and $\left(p_{2}\right.$ $\left.+p_{2}^{\prime}\right)^{\mu}=\left(E+E^{\prime},-\mathbf{q}-\mathbf{q}^{\prime}\right)$. The coupling constants $g_{p s, s, v}$ and $f_{v}$, the cutoff functions $F$ and the meson masses are given in Ref. [15].

In order to bring this OBE potential in a form consistent with our three-dimensional (3D) equations, the bilinear Dirac forms have to be expressed in terms of the operators $W_{i}$ of Eqs. (3.1). The result in form of the Wolfenstein operators of Eq. (3.5) is given in Appendix B.

Another often used modern $N N$ potential is the Argonne potential AV18 (Ref. [16]). It is originally presented in configuration space and has the general form

$$
V(N N)=v^{E M}(N N)+v^{\pi}(N N)+v^{R}(N N) .
$$

Here $v^{E M}(N N)$ represents an electromagnetic part, which we omit in this work. The one-pion-exchange (OPE) part $v^{\pi}(N N)$ is charge dependent and has the standard form. The Yukawa and tensor functions contain exponential cutoffs, thus do not have an analytical Fourier transform to momentum space. The intermediate- and short-range phenomenological part $v^{R}(N N)$ is expressed as a sum of central, tensor, spin-orbit, $L^{2}$, and quadratic spin-orbit pieces (abbreviated as $c, t, l s, l 2, l s 2$, respectively) in different spin $(S)$ and iso spin (T) states:

$$
\begin{aligned}
v_{S T}^{R}(N N)= & v_{S T}^{c}(r) \mathbf{1}+v_{S T}^{t}(r) S_{12}+v_{S T}^{l s}(r) \mathbf{L} \cdot \mathbf{S}+v_{S T}^{l 2}(r) L^{2} \\
& +v_{S T}^{l s 2}(r)(\mathbf{L} \cdot \mathbf{S})^{2}
\end{aligned}
$$

where $S_{12}$ denotes the standard tensor operator. The specific 
form of the radial functions as well as the potential parameters are given in Ref. [16]. For applying this potential in our formulation, we need to perform the transition to momentum space. For the terms contributing to $v^{R}(N N)$ we obtain explicitly

$$
\begin{aligned}
& v_{S T}^{c}\left(\mathbf{q}^{\prime}, \mathbf{q}\right)=\frac{1}{2 \pi^{2}} \int_{0}^{\infty} d r r^{2} j_{0}(\rho r) v_{S T}^{c}(r), \\
& v_{S T}^{t}\left(\mathbf{q}^{\prime}, \mathbf{q}\right)=\frac{1}{2 \pi^{2}}\left(\frac{-3 \boldsymbol{\sigma}_{1} \cdot\left(\mathbf{q}^{\prime}-\mathbf{q}\right) \boldsymbol{\sigma}_{2} \cdot\left(\mathbf{q}^{\prime}-\mathbf{q}\right)}{\rho^{2}}\right. \\
& \left.+\boldsymbol{\sigma}_{1} \cdot \boldsymbol{\sigma}_{2}\right) \int_{0}^{\infty} d r r^{2} j_{2}(\rho r) v_{S T}^{t}(r), \\
& v_{S T}^{l s}\left(\mathbf{q}^{\prime}, \mathbf{q}\right)=\frac{i}{2 \pi^{2}} \mathbf{S} \cdot\left(\mathbf{q} \times \mathbf{q}^{\prime}\right) \frac{1}{\rho} \int_{0}^{\infty} d r r^{3} j_{1}(\rho r) v_{S T}^{l s}(r), \\
& v_{S T}^{l 2}\left(\mathbf{q}^{\prime}, \mathbf{q}\right)=\frac{1}{2 \pi^{2}} \mathbf{q} \cdot \mathbf{q}^{\prime} \frac{2}{\rho} \int_{0}^{\infty} d r r^{3} j_{1}(\rho r) v_{S T}^{l 2}(r) \\
& -\frac{1}{2 \pi^{2}}\left[\mathbf{q}^{\prime 2} \mathbf{q}^{2}\left(1-\gamma^{2}\right)\right] \frac{1}{\rho^{2}} \\
& \times \int_{0}^{\infty} d r r^{4} j_{2}(\rho r) v_{S T}^{l 2}(r), \\
& v_{S T}^{l s 2}\left(\mathbf{q}^{\prime}, \mathbf{q}\right)=\frac{1}{2 \pi^{2}}\left(\mathbf{S}^{2} \mathbf{q} \cdot \mathbf{q}^{\prime}-\mathbf{S} \cdot \mathbf{q} \mathbf{S} \cdot \mathbf{q}^{\prime}\right) \frac{1}{\rho} \\
& \times \int_{0}^{\infty} d r r^{3} j_{1}(\rho r) v_{S T}^{l s 2}(r)-\frac{1}{2 \pi^{2}}\left(\frac{1}{2}\left(\mathbf{q} \times \mathbf{q}^{\prime}\right)^{2}\right. \\
& \left.+\frac{1}{2} \boldsymbol{\sigma}_{1} \cdot\left(\mathbf{q} \times \mathbf{q}^{\prime}\right) \boldsymbol{\sigma}_{2} \cdot\left(\mathbf{q} \times \mathbf{q}^{\prime}\right)\right) \frac{1}{\rho^{2}} \\
& \times \int_{0}^{\infty} d r r^{4} j_{2}(\rho r) v_{S T}^{l s 2}(r)
\end{aligned}
$$

where $\rho \equiv\left|\mathbf{q}^{\prime}-\mathbf{q}\right|$. The resulting operators can be easily rep- resented as function of the Wolfenstein operators $W_{i}$. The final expression are given in Appendix C.

\section{CONNECTION TO A PARTIAL WAVE REPRESENTATION}

In order to compare with standard partial representations, especially $N N$ phase shifts, we need to make connection to the standard partial wave representation. The partial wave projected $T$-matrix element is defined as

$$
T_{l^{\prime} l}^{S j t}(q) \equiv\left\langle q\left(l^{\prime} S\right) j m t m_{t}|T| q(l S) j m t m_{t}\right\rangle,
$$

where the states $\left|q(l S) j m t m_{t}\right\rangle$ are given as

$$
\left|q(l S) j m t m_{t}\right\rangle=\sum_{\mu} C(l S j, \mu m-\mu)|q l \mu\rangle|S m-\mu\rangle\left|t m_{t}\right\rangle .
$$

We choose the standard normalization for those states, namely

$$
\begin{aligned}
& \left\langle q^{\prime}\left(l^{\prime} S^{\prime}\right) j^{\prime} m^{\prime} t^{\prime} m_{t}^{\prime} \mid q(l S) j m t m_{t}\right\rangle \\
& =\frac{\delta(q-q)}{q^{\prime} q} \delta_{l^{\prime} l} \delta_{S^{\prime} S} \delta_{j^{\prime} j} \delta_{m^{\prime} m} \delta_{t^{\prime} t} \delta_{m_{t}^{\prime} m_{t}} .
\end{aligned}
$$

The connection to the states $|\mathbf{q} ; \hat{q} S \Lambda\rangle$ can be found using Eq. (2.9), and one finds

$$
\begin{aligned}
|\mathbf{q} ; \hat{q} S \Lambda\rangle= & \sum_{l j m}|q(l S) j m\rangle \sum_{\mu} C(l S j ; \mu m-\mu) Y_{l \mu}^{*}(\hat{q}) \\
& \times e^{-i(m-\mu) \phi} d_{m-\mu, \Lambda}^{S}(\theta) .
\end{aligned}
$$

Consequently one obtains for the $T$ matrix

$$
\begin{aligned}
T_{\Lambda^{\prime} \Lambda}^{\pi S t}\left(\mathbf{q}^{\prime}, \mathbf{q}\right)= & \sqrt{2}\left[1-\eta_{\pi}(-)^{S+t}\right]_{\pi}\left\langle\mathbf{q}^{\prime} ; \hat{q}^{\prime} S \Lambda^{\prime}|\langle t|T| t\rangle| \mathbf{q}\right\rangle|\hat{q} S \Lambda\rangle \\
= & \frac{1}{2}\left[1-\eta_{\pi}(-)^{S+t}\right] \sum_{l^{\prime} l j m} T_{l^{\prime} l}^{S j t}(q)\left[1+\eta_{\pi}(-)^{l^{\prime}}\right]\left[1+\eta_{\pi}(-)^{l}\right] \sum_{\mu^{\prime}} C\left(l^{\prime} S j ; \mu^{\prime}, m-\mu^{\prime}\right) \\
& \times Y_{l^{\prime} \mu^{\prime}}\left(\hat{q}^{\prime}\right) e^{i\left(m-\mu^{\prime}\right) \phi^{\prime}} d_{m-\mu^{\prime}, \Lambda^{\prime}}^{S}\left(\theta^{\prime}\right) \sum_{\mu} C(l S j ; \mu, m-\mu) Y_{l \mu}^{*}(\hat{q}) e^{-i(m-\mu) \phi} d_{m-\mu, \Lambda}^{S}(\theta)
\end{aligned}
$$

For $\mathbf{q}$ parallel to the $z$ axis, i.e., $\hat{q}=\hat{z}$, one finds after some straightforward algebra the on-shell relation 


$$
\begin{aligned}
T_{\Lambda^{\prime} \Lambda}^{\pi S t}\left(q, q, \theta^{\prime}\right)= & \frac{1}{2}\left[1-\eta_{\pi}(-)^{S+t}\right] \sum_{l^{\prime} l j} T_{l^{\prime} l}^{S j t}(q)\left[1+\eta_{\pi}(-)^{l^{\prime}}\right] \\
& \times\left[1+\eta_{\pi}(-)^{l}\right] \sqrt{\frac{2 l^{\prime}+1}{4 \pi}} C\left(l^{\prime} S j ; 0 \Lambda^{\prime}\right) d_{\Lambda \Lambda^{\prime}}^{j}\left(\theta^{\prime}\right) \sqrt{\frac{2 l+1}{4 \pi}} C(l S j ; 0 \Lambda) .
\end{aligned}
$$

Here the relation

$$
\begin{aligned}
Y_{l^{\prime} \mu^{\prime}}\left(\hat{q}^{\prime}\right) & =\sqrt{\frac{2 l^{\prime}+1}{4 \pi}} D_{\mu^{\prime}, 0}^{l^{\prime} *}\left(\phi^{\prime} \theta^{\prime} 0\right) \\
& =\sqrt{\frac{2 l^{\prime}+1}{4 \pi}}(-)^{\mu^{\prime}} D_{-\mu^{\prime}, 0}^{l^{\prime}}\left(\phi^{\prime} \theta^{\prime} 0\right)
\end{aligned}
$$

was used together with an addition theorem for $D$ functions.

Once the partial wave projected $T$ matrices are calculated, we can use the the well-known connection to the partial wave $S$ matrices,

$$
S_{l^{\prime} l}^{S j t}=\delta_{l^{\prime} l}-\pi i m q T_{l^{\prime} l}^{S j t},
$$

which are parametrized by the standard partial wave phase shifts.

The inversion of Eq. (5.6) can be accomplished by using the orthogonality relation

$$
\int_{0}^{2 \pi} d \phi \int_{0}^{\pi} d \theta \sin \theta D_{\mu m}^{j_{1}^{*}}(\phi \theta 0) D_{\mu m}^{j_{2}}(\phi \theta 0)=\frac{4 \pi}{2 j_{1}+1} \delta_{j_{1} j_{2}}
$$

In addition we take into account Eq. (3.14) for the on-shell $T$ matrix and find after a few steps

$$
\begin{aligned}
& T_{l^{\prime} l}^{S j t}(q)=4 \pi\left[1-\eta_{\pi}(-)^{S+t}\right]^{-1}\left[1+\eta_{\pi}(-)^{l^{\prime}}\right]^{-1} \\
& \times\left[1+\eta_{\pi}(-)^{l}\right]^{-1} \frac{\sqrt{2 l^{\prime}+1} \sqrt{2 l+1}}{2 j+1} \\
& \times \sum_{\Lambda^{\prime} \Lambda} C\left(l^{\prime} S j ; 0 \Lambda^{\prime}\right) C(l S j ; 0 \Lambda) \int_{-1}^{1} d\left(\cos \theta^{\prime}\right) d_{\Lambda \Lambda^{\prime}}^{j}\left(\theta^{\prime}\right) \\
& \times T_{\Lambda^{\prime} \Lambda}^{\pi S t}\left(q, q, \theta^{\prime}\right) .
\end{aligned}
$$

Finally we connect the physical $T$-matrix element to the partial wave projected $S$ matrices. We insert Eq. (5.6) into Eq. (2.28). In proceeding we use

$$
D_{\Lambda \Lambda^{\prime}}^{j *}\left(\phi^{\prime} \theta^{\prime} 0\right)=(-)^{\Lambda-\Lambda^{\prime}} e^{i \Lambda \phi^{\prime}} d_{-\Lambda-\Lambda^{\prime}}^{j}\left(\theta^{\prime}\right)
$$

and an addition theorem of $D$ functions together with Eq. (5.7). Then we end up with the standard form

$$
\begin{aligned}
& { }_{a}\left\langle\nu_{1} \nu_{2} m_{1}^{\prime} m_{2}^{\prime} q \hat{q}^{\prime}|T| \nu_{1} \nu_{2} m_{1} m_{2} \vec{q}\right\rangle_{a} \\
& =\frac{1}{2} \frac{1}{\sqrt{4 \pi}} \frac{1}{\pi i m q} \sum_{S t} C\left(\frac{1}{2} \frac{1}{2} t, \nu_{1} \nu_{2}\right)^{2} \\
& \times C\left(\frac{1}{2} \frac{1}{2} S, m_{1}^{\prime} m_{2}^{\prime} \Lambda_{0}^{\prime}\right) C\left(\frac{1}{2} \frac{1}{2} S, m_{1} m_{2} \Lambda_{0}\right) \\
& \times \sum_{j l^{\prime} l}\left[1-(-)^{l+S+t}\right]\left[1-(-)^{l^{\prime}+S+t}\right] \\
& \times\left(\delta_{l l^{\prime}}-S_{l^{\prime} l}^{S j t}\right) \sqrt{2 l+1} C\left(l S j, 0 \Lambda_{0}\right) \\
& \times C\left(l^{\prime} S j, \Lambda_{0}-\Lambda_{0}^{\prime}, \Lambda_{0}^{\prime}\right) Y_{l^{\prime}, \Lambda_{0}-\Lambda_{0}^{\prime}}\left(\theta^{\prime}, \phi^{\prime}\right) \text {. }
\end{aligned}
$$

One can choose $\phi^{\prime}=0$ by putting $\mathbf{q}^{\prime}$ into the $x-z$ plane.

In order to unambiguously define the normalization we also give the expression for the spin-averaged differential cross section for nucleon species $\nu_{1} \nu_{2}$ as

$$
\begin{aligned}
\frac{d \sigma}{d \Omega}= & (2 \pi)^{4}\left(\frac{m}{2}\right)^{2} \frac{1}{4} \\
& \times \sum_{m_{1}^{\prime} m_{2}^{\prime} m_{1} m_{2}}\left|{ }_{a}\left\langle\nu_{1} \nu_{2} m_{1}^{\prime} m_{2}^{\prime} q \hat{q}^{\prime}|T| \nu_{1} \nu_{2} m_{1} m_{2} \mathbf{q}\right\rangle_{a}\right|^{2} .
\end{aligned}
$$

\section{RESULTS AND DISCUSSION}

In order to demonstrate the feasibility of our formulation when applied to $N N$ scattering, we present numerical results for two $N N$ potentials of quite different character, the Bonn-B (Ref. [15]) and the AV18 (Ref. [16]) potential models. For calculating the $N N t$ matrix we solve the integral equation, Eq. (3.17). For $S=0$ this is a single, two dimensional integral equation in two variables, for the parity even and odd part, respectively. For $S=1$ there are two sets of coupled integral equations in two variables for the helicities $\Lambda=0,1$. The $\phi^{\prime \prime}$ integration over the potential, Eq. (3.18), can be carried out independently, and thus does not enter the integral equation as separate variable. As it turns out, both potentials only depend weakly on this angle, and one typically needs ten integration points in the case of Bonn-B and 16 in the case of AV18 to have sufficient accuracy. Typical grids needed for the $\theta^{\prime \prime}$ integration consist of 32-48 points and for the $q^{\prime \prime}$ integration of 48-72 points, depending on the desired accuracy. 
TABLE I. Comparison of the nucleon-nucleon phase shifts calculated from our three dimensional formulation (helicitiy) with a standard partial wave calculation (carried out in momentum space) for the Bonn-B potential at 100 and $300 \mathrm{MeV}$ laboratory energy.

\begin{tabular}{|c|c|c|c|c|}
\hline \multirow[b]{2}{*}{${ }^{2 S+1} \mathrm{~L}_{J}$} & \multicolumn{2}{|c|}{$E_{l a b}=100 \mathrm{MeV}$} & \multicolumn{2}{|c|}{$E_{l a b}=300 \mathrm{MeV}$} \\
\hline & 3D helicity & Partial wave & 3D helicity & Partial wave \\
\hline${ }^{1} S_{0}$ & 25.1928 & 25.1929 & -8.1755 & -8.1756 \\
\hline${ }^{3} P_{0}$ & 9.8046 & 9.8046 & -11.4799 & -11.4799 \\
\hline${ }^{1} P_{1}$ & -16.3131 & -16.3126 & -28.6946 & -28.6922 \\
\hline${ }^{3} P_{1}$ & -13.4677 & -13.4677 & -26.3800 & -26.3800 \\
\hline${ }^{3} S_{1}$ & 41.9858 & 41.9870 & 4.0667 & 4.0676 \\
\hline${ }^{3} D_{1}$ & -12.9847 & -12.9846 & -23.7182 & -23.7181 \\
\hline$\varepsilon_{1}$ & -2.2360 & -2.2357 & -4.0268 & -4.0265 \\
\hline${ }^{1} D_{2}$ & 3.3411 & 3.3411 & 7.4888 & 7.4888 \\
\hline${ }^{3} D_{2}$ & 17.6710 & 17.6710 & 25.3616 & 25.3617 \\
\hline${ }^{3} P_{2}$ & 11.7356 & 11.7356 & 17.3981 & 17.3981 \\
\hline${ }^{3} F_{2}$ & 0.7705 & 0.7705 & 0.5236 & 0.5238 \\
\hline$\varepsilon_{2}$ & 2.8402 & 2.8402 & 2.0166 & 2.0166 \\
\hline${ }^{1} F_{3}$ & -2.4397 & -2.4397 & -5.5865 & -5.5865 \\
\hline${ }^{3} F_{3}$ & -1.6484 & -1.6484 & -4.0097 & -4.0097 \\
\hline${ }^{3} D_{3}$ & 0.4203 & 0.4855 & 2.5719 & 2.5720 \\
\hline${ }^{3} G_{3}$ & -1.0105 & -1.0105 & -4.4051 & -4.4051 \\
\hline$\varepsilon_{3}$ & -3.6604 & -3.6604 & -7.2233 & -7.2233 \\
\hline${ }^{1} G_{4}$ & 0.4092 & 0.4092 & 1.3556 & 1.3556 \\
\hline${ }^{3} G_{4}$ & 2.2624 & 2.2624 & 7.3000 & 7.3000 \\
\hline${ }^{3} F_{4}$ & 0.4203 & 0.4203 & 2.4491 & 2.4491 \\
\hline${ }^{3} \mathrm{H}_{4}$ & 0.1082 & 0.1082 & 0.5077 & 0.5077 \\
\hline$\varepsilon_{4}$ & 0.5575 & 0.5575 & 1.5509 & 1.5509 \\
\hline
\end{tabular}

The Cauchy singularity in Eq. (3.17) is separated into a principal value part and a $\delta$-function part, and the principal value singularity is treated by subtraction. In case of the Bonn-B potential the integration interval for the $q^{\prime \prime}$ integration is covered by mapping the Gauss-Legendre points $u$ from the interval $(0,1)$ via

$$
q=b \tan \left(\frac{\pi}{2} u\right)
$$

to the interval $(0, \infty)$. Typical values of $b$ are $1000 \mathrm{MeV} / c$. For the AV18 potential this type of integration map is less useful due to the difficulty in handling the numerical Fourier transform for very large values of $q^{\prime \prime}$, and the integration interval is cut off at $150 \mathrm{fm}^{-1}$, which is according to our experience sufficient. The Fourier-Bessel transform of the AV18 potential functions are carried out using Filon's quadrature formula [21], which proves to be more accurate in handling the strong oscillatory behavior of the integrands in Eqs. (4.6)-(4.10) for large values of the integration parameter $r$, compared to, e.g., Simpson's rule.

A stringent test for our numerics is a comparison of the $N N$ phase shifts, which we obtain from Eq. (5.10) together
TABLE II. Comparison of the nucleon-nucleon phase shifts calculated from our three dimensional formulation (helicity) with a standard partial wave calculation (carried out in coordinate space [22]) for the AV18 potential at 100 and $300 \mathrm{MeV}$ laboratory energy.

\begin{tabular}{|c|c|c|c|c|}
\hline \multirow[b]{2}{*}{${ }^{2 S+1} \mathrm{~L}_{J}$} & \multicolumn{2}{|c|}{$E_{l a b}=100 \mathrm{MeV}$} & \multicolumn{2}{|c|}{$E_{l a b}=300 \mathrm{MeV}$} \\
\hline & 3D helicity & Partial wave & 3D helicity & Partial wave \\
\hline${ }^{1} S_{0}$ & 25.99 & 25.94 & -4.62 & -4.60 \\
\hline${ }^{3} P_{0}$ & 8.69 & 8.69 & -11.05 & -11.06 \\
\hline${ }^{1} P_{1}$ & -14.19 & -14.20 & -26.18 & -26.28 \\
\hline${ }^{3} P_{1}$ & -13.06 & -13.07 & -28.38 & -28.49 \\
\hline${ }^{3} S_{1}$ & 43.69 & 43.56 & 8.15 & 8.16 \\
\hline${ }^{3} D_{1}$ & -12.08 & -12.09 & -24.80 & -24.90 \\
\hline$\varepsilon_{1}$ & -2.49 & -2.49 & -4.38 & -4.39 \\
\hline${ }^{1} D_{2}$ & 3.81 & 3.81 & 9.45 & 9.44 \\
\hline${ }^{3} D_{2}$ & 17.14 & 17.10 & 25.11 & 25.02 \\
\hline${ }^{3} P_{2}$ & 11.02 & 11.00 & 16.96 & 16.91 \\
\hline${ }^{3} F_{2}$ & 0.67 & 0.67 & 0.77 & 0.76 \\
\hline$\varepsilon_{2}$ & 2.70 & 2.70 & 2.21 & 2.21 \\
\hline${ }^{1} F_{3}$ & -2.23 & -2.23 & -4.87 & -4.88 \\
\hline${ }^{3} F_{3}$ & -1.35 & -1.35 & -2.51 & -2.51 \\
\hline${ }^{3} D_{3}$ & 1.61 & 1.61 & 5.22 & 5.21 \\
\hline${ }^{3} G_{3}$ & -0.93 & -0.93 & -4.19 & -4.20 \\
\hline$\varepsilon_{3}$ & -3.50 & -3.50 & -7.17 & -7.16 \\
\hline${ }^{1} G_{4}$ & 0.40 & 0.40 & 1.42 & 1.42 \\
\hline${ }^{3} G_{4}$ & 2.22 & 2.22 & 7.35 & 7.34 \\
\hline${ }^{3} F_{4}$ & 0.45 & 0.45 & 2.75 & 2.74 \\
\hline${ }^{3} H_{4}$ & 0.07 & 0.07 & 0.31 & 0.31 \\
\hline$\varepsilon_{4}$ & 0.51 & 0.51 & 1.54 & 1.54 \\
\hline
\end{tabular}

with Eq. (5.8), with phase shifts calculated with standard partial wave techniques. The result for the Bonn-B phase shifts is given in Table I for projectile energies 100 and 300 $\mathrm{MeV}$. The agreement of both calculations is excellent. In Table II the equivalent comparison is given between the phase shifts calculated from the AV18 potential with our 3D formulation and a standard partial wave calculation (in this case in coordinate space). The agreement is also very good, however, not as perfect as in the Bonn-B case. The reason for this slight discrepancy is presumably twofold. First, we carry out a numerical Fourier-Bessel transformation of the different potential functions of AV18. The solution of the $t$-matrix integral equation requires an evaluation of the potential function on a grid of the size $n_{\phi^{\prime \prime}} \times n_{\theta^{\prime \prime}} \times n_{q^{\prime \prime}} \times n_{q^{\prime}} \times n_{\theta^{\prime}}$. For computational economy we calculate the potential functions on a fine grid for $\rho=\mid \mathbf{q}^{\prime \prime}$ $-\mathbf{q} \mid$ and obtain the points actually needed in the calculation via interpolation. This procedure naturally leads to larger numerical errors compared to a direct evaluation of algebraic expressions as is the case for Bonn-B. The differences can be clearly seen when comparing Tables I and II. In both cases a comparable grid for the $t$ matrices is used. 

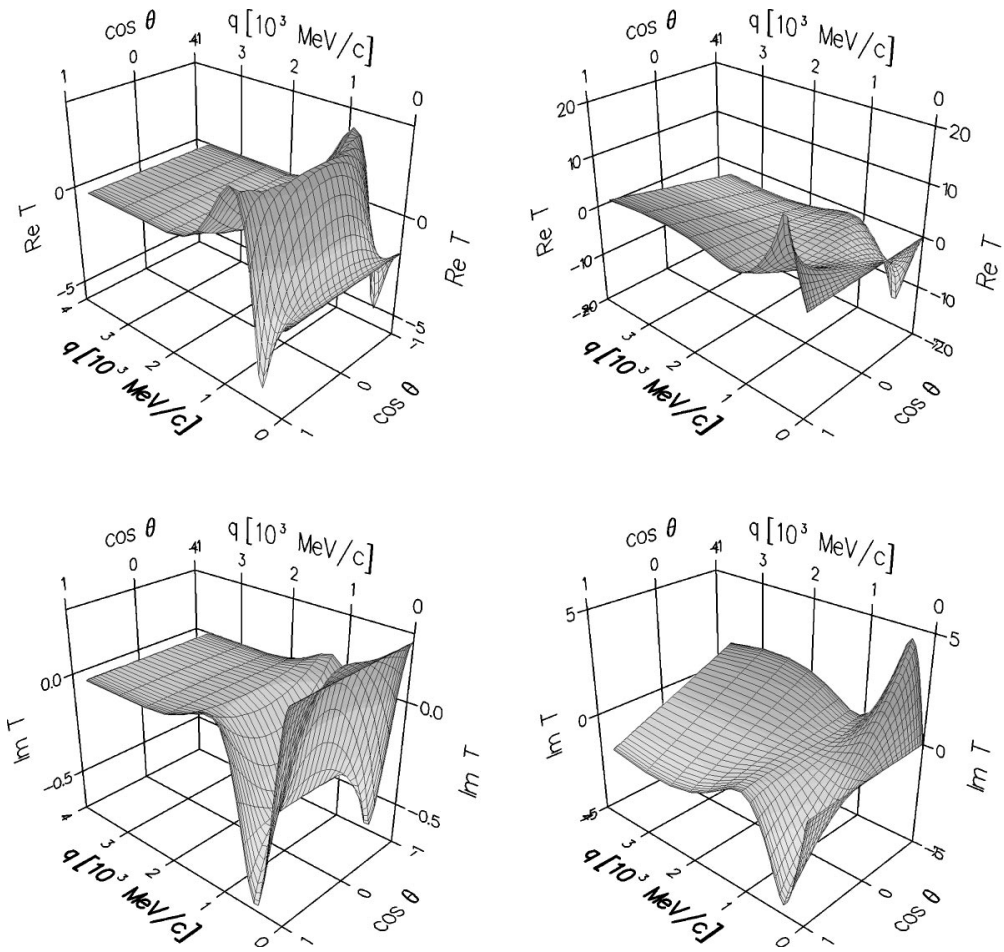

FIG. 1. The half-shell $t$ matrix $T_{\Lambda^{\prime} \Lambda}^{\pi S t}\left(q, q_{0}, \theta\right)$ for $q_{0}=375 \mathrm{MeV} / c$, corresponding to $E_{l a b}=300$ $\mathrm{MeV}$, calculated from the Bonn-B potential. The left side displays the real and imaginary parts of $T_{00}^{101}\left(q, q_{0}, \theta\right)$ as function of $q$ and $x=\cos \theta$. The right side shows $T_{00}^{-100}\left(q, q_{0}, \theta\right)$. The units of $T_{00}^{\pi 0 t}\left(q, q_{0}, \theta\right)$ are $10^{-7} \mathrm{MeV}^{-2}$.

Next we want to display the angular and momentum dependence of the half-shell $t$ matrices $T_{\Lambda^{\prime} \Lambda}^{\pi S t}\left(q, q_{0}, \theta\right)$ as generated from Eq. (3.17). For the case $S=0$ this is done in Fig. 1 for the Bonn-B potential for the parity-even (left side) and for the parity-odd case (right side). The parity-even $t$ matrix exhibits a similar behavior to the symmetrized $t$ matrix of the two-boson case studied in Ref. [6]. It shows strong forward and backward peaks around the on-shell momentum $q_{0}$. Once far away from $q_{0}$ the momentum dependence is quite moderate. The parity-odd $t$ matrix also displays the the strong peaks around the on-shell momentum, however, the forward and backward peaks have opposite signs.

For the case $S=1$ there are two coupled integral equations as shown in Eq. (3.17). Due to the symmetries of the
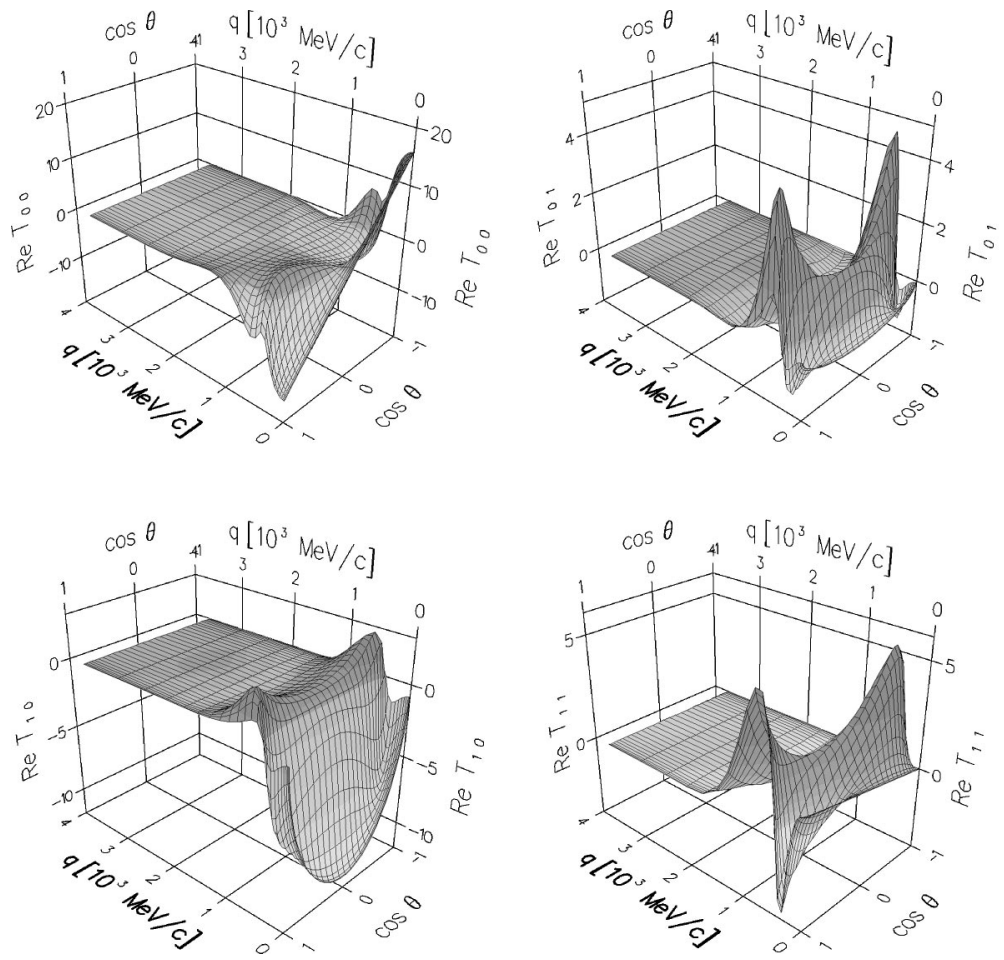

FIG. 2. The real part of the parity-even halfshell $t$ matrix $T_{\Lambda^{\prime} \Lambda_{\Lambda}}^{110}\left(q, q_{0}, \theta\right)$ for $q_{0}=375 \mathrm{MeV} / \mathrm{c}$. The helicities $\Lambda^{\prime}, \Lambda=0,1$ are kept as subscripts of $T$. All $t$ matrices are calculated using the Bonn-B potential and are given in units $10^{-7} \mathrm{MeV}^{-2}$. 

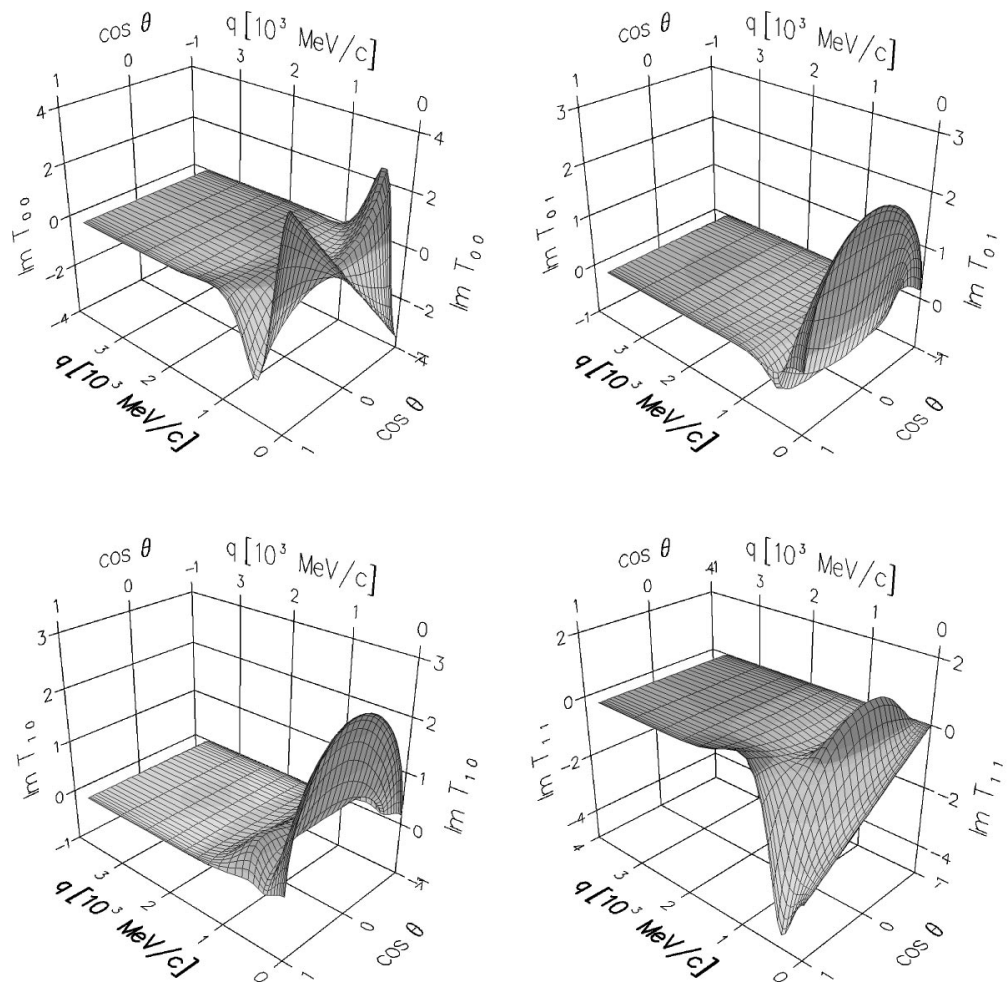

FIG. 3. Same as Fig. 2, but for the imaginary part of $T_{\Lambda^{\prime} \Lambda}^{110}\left(q, q_{0}, \theta\right)$.

potential, Eq. (2.19), it is sufficient to consider only the helicity combinations $\Lambda^{\prime}, \Lambda=0,1$. The corresponding half-shell $t$ matrices, $T_{\Lambda^{\prime} \Lambda}^{\pi 1 t}\left(q, q_{0}, \theta\right)$ obtained from the Bonn-B potential are displayed in Figs. 2-5. The real and imaginary parts show a rich structure as function of angle and for momenta smaller than $1000 \mathrm{MeV} / c$. For momenta larger than 1000 $\mathrm{MeV} / \mathrm{c}$ all amplitudes show no or only a very weak angular dependence. We would like to point out that at $300 \mathrm{MeV}$ projectile energy partial waves have to be summed at least to $J=6$ to obtain a converged result for calculating, e.g., observables.

A further question of interest is how strong the difference is between the half-shell $t$-matrix amplitudes derived from the two potentials presented here. In Fig. 6 the real and
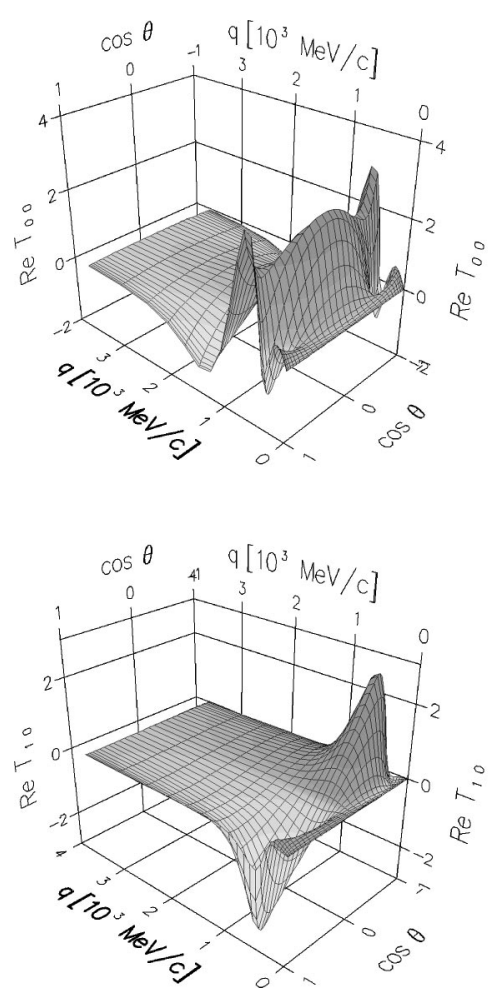
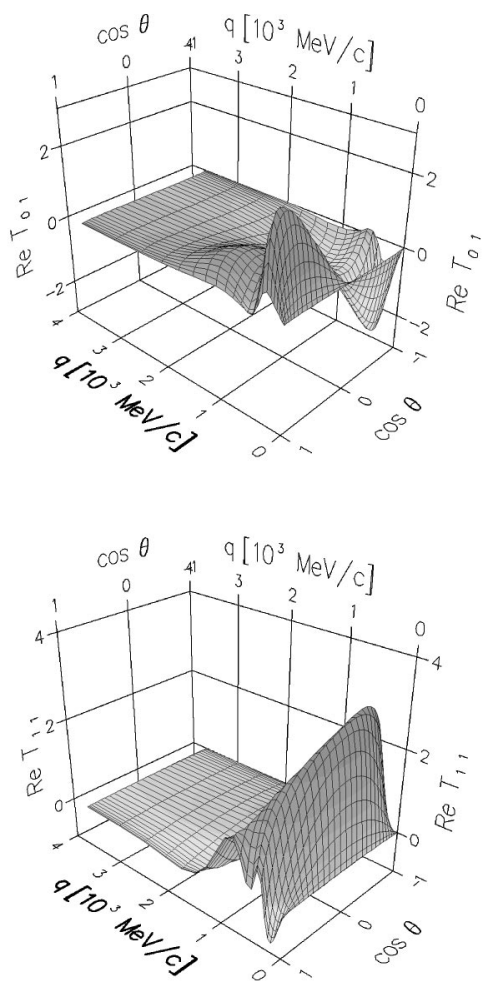

FIG. 4. The real part of the half-shell parityodd half-shell $t$ matrix $T_{\Lambda^{\prime} \Lambda}^{-111}\left(q, q_{0}, \theta\right)$ for $q_{0}$ $=375 \mathrm{MeV} / \mathrm{c}$ calculated from the Bonn-B potential. The description is the same as in Fig. 2. 

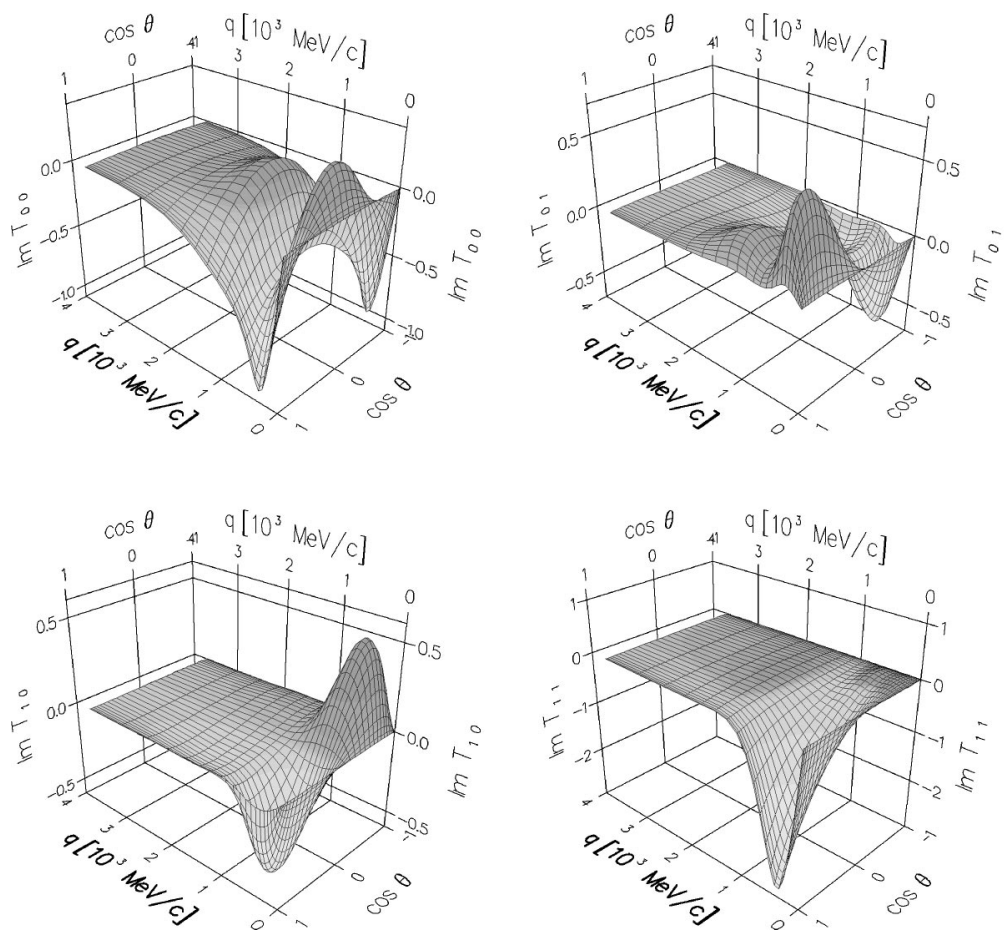

FIG. 5. Same as Fig. 4, but for the imaginary part of $T_{\Lambda^{\prime} \Lambda}^{-111}\left(q, q_{0}, \theta\right)$.

imaginary parts of $T_{\Lambda^{\prime} \Lambda}^{\pi S t}\left(q, q_{0}, \theta\right)$ for $S=0$ calculated with AV18 are shown. Of course along the line $q=q_{0}$ the corresponding amplitudes derived from the two potentials are identical. However, if one compares the detailed structures of especially the parity odd amplitudes, one sees that at large momenta $q$ the Bonn-B amplitude shows more structure than the one derived from AV18. For the case $S=1$ we only want to show a selection of $t$-matrix amplitudes calculated from AV18. In general one can say that some amplitudes are very similar to the ones derived from Bonn-B, others are quite different. The choices displayed in Fig. 7 are being representative. The upper two $t$ matrices show a considerable difference to the corresponding ones of Bonn-B, while the lower ones are relatively similar.

Next we turn to the on-the-energy-shell $t$-matrix amplitudes as given in Eq. (2.28) in terms of the $T_{\Lambda^{\prime} \Lambda}^{\pi S t}$ quantities. Here it is interesting to consider $\left|{ }_{a}\left\langle\nu_{1} \nu_{2} m_{1}^{\prime} m_{2}^{\prime} q_{0} \hat{q}|T| \nu_{1} \nu_{2} m_{1} m_{2} \mathbf{q}_{0}\right\rangle_{a}\right|^{2} \equiv\left|\left\langle m_{1}^{\prime} m_{2}^{\prime}|T| m_{1} m_{2}\right\rangle\right|^{2}$,
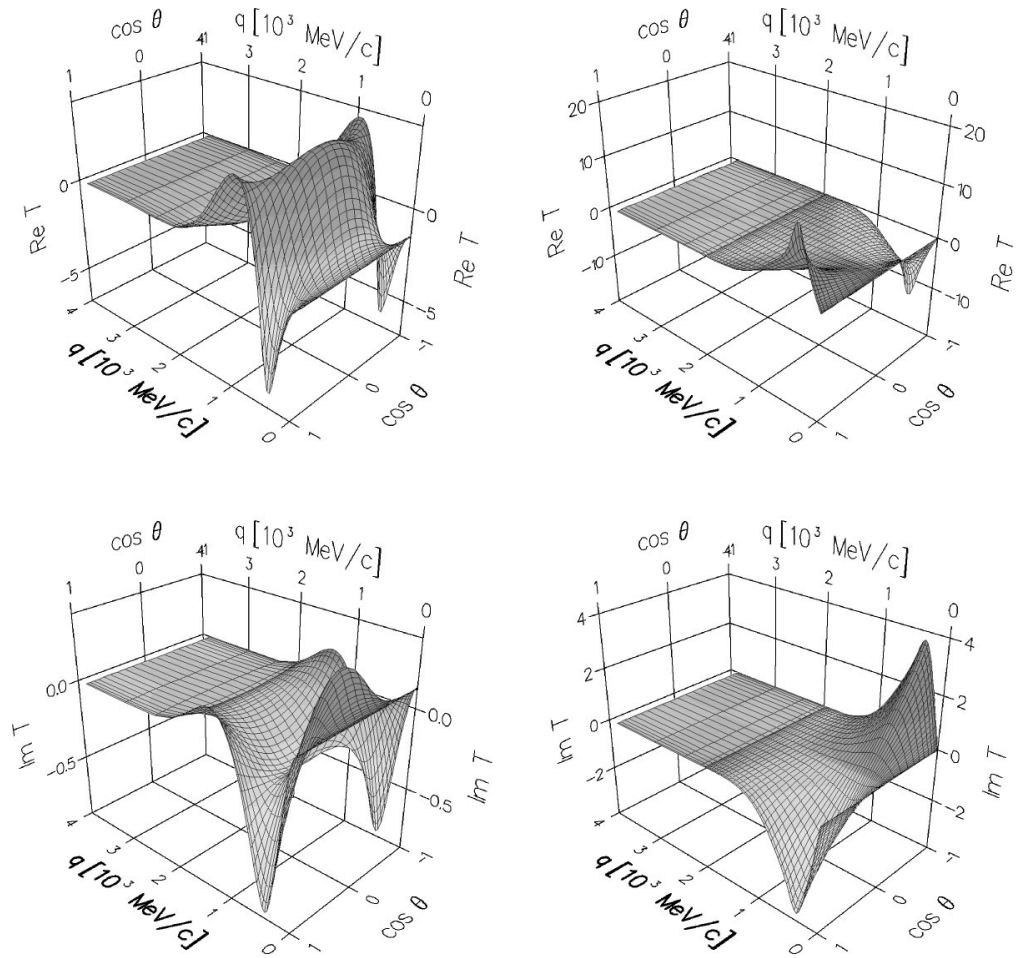

044002-12
FIG. 6. The half-shell $t$ matrix $T_{\Lambda^{\prime}{ }^{\prime} \Lambda}^{\pi 0 t}\left(q, q_{0}, \theta\right)$ for $q_{0}=375 \mathrm{MeV} / c$ calculated from the AV18 potential. The left side displays the real and imaginary parts of $T_{00}^{101}\left(q, q_{0}, \theta\right)$ as function of $q$ and $x=\cos \theta$. The right side shows $T_{00}^{-100}\left(q, q_{0}, \theta\right)$. The units of $T_{00}^{\pi 0 t}\left(q^{\prime}, q_{0}, \theta\right)$ are $10^{-7} \mathrm{MeV}^{-2}$. 

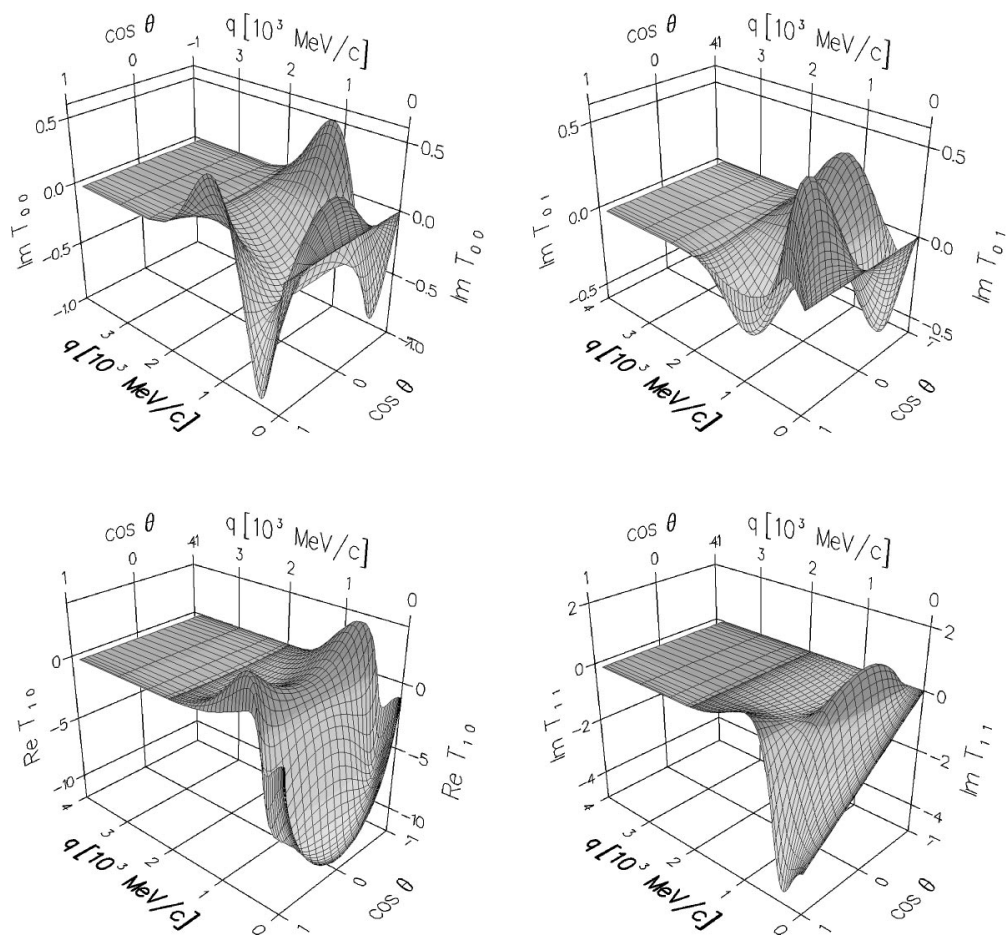

FIG. 7. Selected half-shell $t$ matrices $T_{\Lambda^{\prime} \Lambda}^{\pi 1 t}\left(q, q_{0}, \theta\right)$ for $q_{0}=375 \mathrm{MeV} / c$ calculated from the AV18 potential. The helicities $\Lambda^{\prime}, \Lambda$ $=0,1$ are kept as subscripts of $T$, which is given in units of $10^{-7} \mathrm{MeV}^{-2}$. where $m= \pm \frac{1}{2}$. If one takes rotational symmetry and parity invariance into account, one ends up with six independent amplitudes,

$$
\begin{gathered}
\langle++|T|++\rangle=\langle--|T|--\rangle, \\
\langle++|T|--\rangle=\langle--|T|++\rangle, \\
\langle+-|T|++\rangle=\langle-+|T|++\rangle=-\langle+-|T|--\rangle \\
=-\langle-+|T|--\rangle, \\
\langle++|T|+-\rangle=\langle++|T|-+\rangle=-\langle--|T|+-\rangle \\
=-\langle--|T|-+\rangle, \\
\langle+-|T|+-\rangle=\langle-+|T|-+\rangle, \\
\langle-+|T|+-\rangle=\langle+-|T|-+\rangle .
\end{gathered}
$$

The six amplitudes given above are displayed in Figs. 8-10 for Bonn-B as function of energy and c.m. scattering angle $\cos \theta$. Though both potentials are only meant to be applied below the pion production threshold, we show the on-shell amplitudes up to $1 \mathrm{GeV}$. Since we do not work with partial waves, a calculation at higher energies takes the same effort as one at very low energies. The sum over all indices $m_{i}$ of the on-shell amplitudes shown gives the spin averaged differential cross section as indicated in Eq. (5.13). We would like to remark that these on-shell amplitudes can also be obtained from the partial wave projected $S$-matrix elements as indicated in Eq. (5.12). We used this relation for numerical tests of our formulation.

\section{SUMMARY}

Two nucleon scattering at intermediate energies of a few hundred $\mathrm{MeV}$ requires quite a few angular momentum states in order to achieve convergence of, e.g., scattering observ-
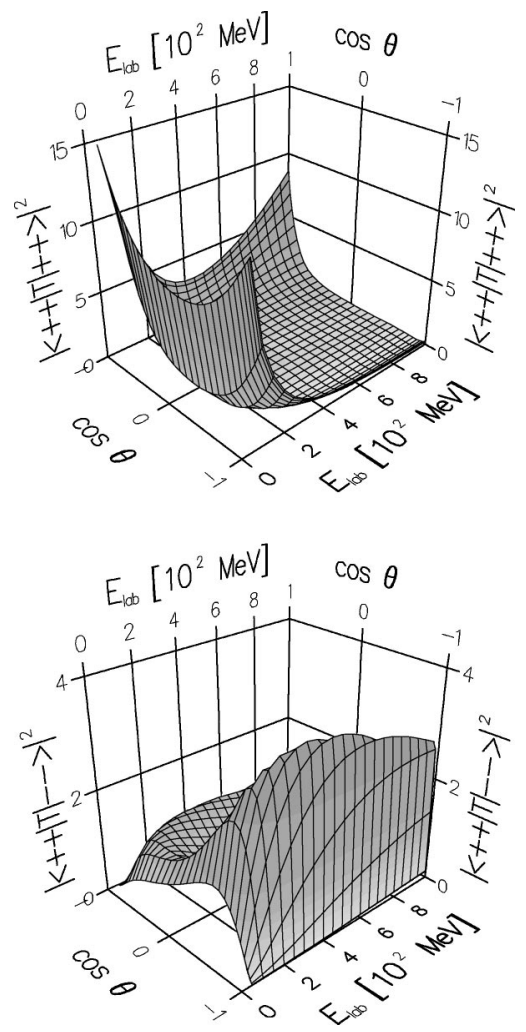

FIG. 8. The absolute value squared of the physical on-shell $T$ matrix elements $\left|\left\langle m_{1}^{\prime} m_{2}^{\prime}|T| m_{1} m_{2}\right\rangle\right|^{2}$ in units $10^{-14} \mathrm{MeV}^{-4}$. The values of $m= \pm \frac{1}{2}$ are abbreviated as \pm . 

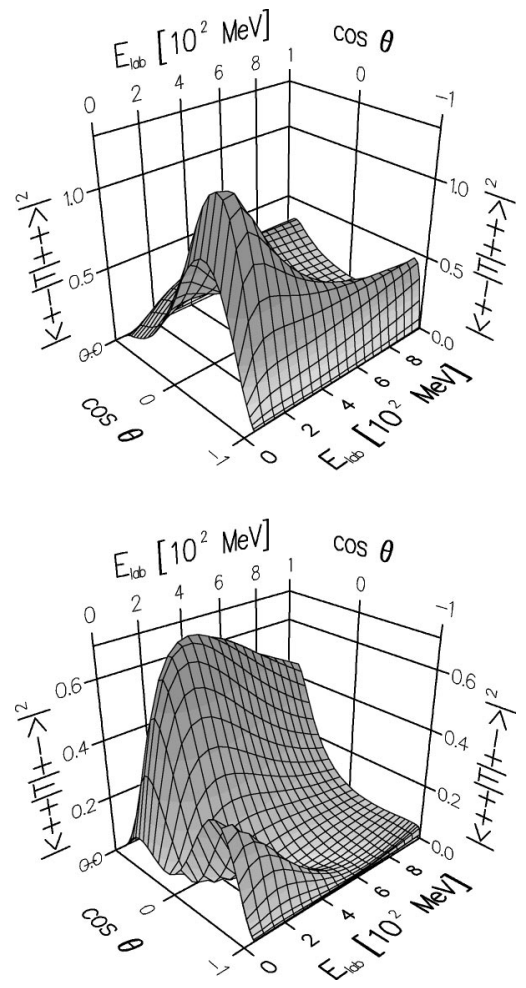

FIG. 9. Same as Fig. 8, but for different $m$ combinations.

ables. This is even more true for the scattering of three or more nucleons upon each other. An alternative approach to the conventional one, which is based on angular momentum decomposition, is to work directly with momentum vectors, specifically with the magnitudes of the momenta and the angles between them. We formulated and numerically illustrated this alternative approach for the case of $N N$ scattering using two realistic interaction models. The momentum vectors enter directly into the scattering equation, and the total spin of the two nucleons is treated in a helicity representation with respect to the relative momenta of the two nucleons. Using rotational and parity invariance one finds in the triplet case $S=1$ a set of two coupled Lippmann-Schwinger equations for each parity and each initial helicity state. Because of symmetry properties only two of the originally three initial helicity states need to be considered. In the singlet case $S=0$ there is only one single Lippmann-Schwinger equation for each parity. The Lippmann-Schwinger equations (uncoupled and coupled) are two dimensional integral equations in two variables for the half-shell $t$ matrix and three variables for the fully off-shell $t$ matrix, namely two magnitudes of momenta and one angle. Though we start with a three dimensional integration, one angle can be integrated out independently, so that we are left with a two dimensional integral equation.

A formulation without angular momentum decomposition is best suited for interaction models which are given in an operator form. In this work we considered the Bonn-B and the AV18 potentials. The helicity representation of the potentials is given in the six linear operators $\Omega_{i}$ of Eq. (3.6), depending on spin and momenta, which are most suited for our formulation. For completeness we also give the poten-
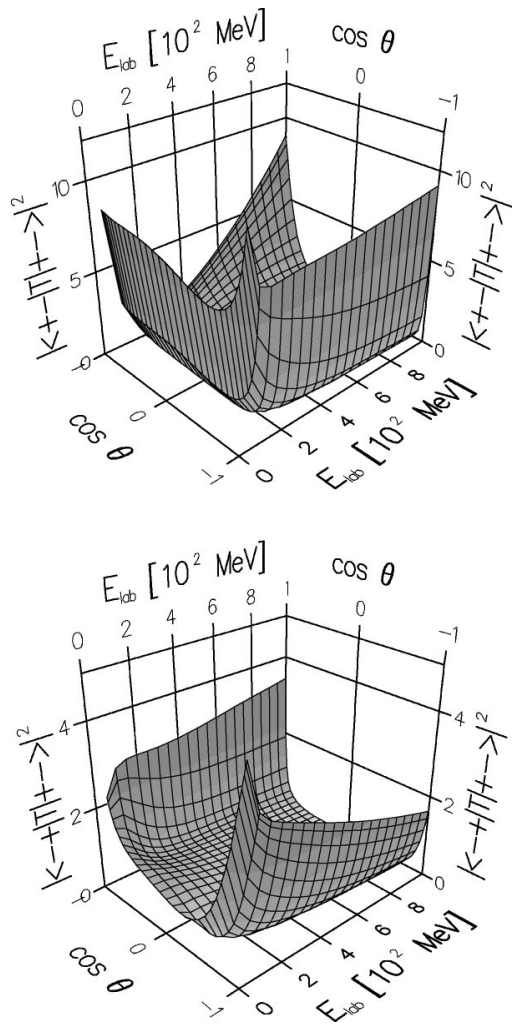

FIG. 10. Same as Fig. 8, but for different $m$ combinations.

tials represented via Wolfenstein operators. We demonstrate the feasibility and accuracy of our three dimensional formulation by projecting the on-shell $t$-matrix elements on angular momentum states and compare the resulting phase shifts with those obtained from standard partial wave projected Lippmann-Schwinger equations. The agreement is very good and demonstrates the numerical reliability and accuracy of our method.

The complete set of half-shell $t$-matrix amplitudes at 300 $\mathrm{MeV}$ laboratory energy is displayed for the Bonn-B potential to give an impression of their rich structure in momentum and angle variables. Nevertheless, the resulting angular dependence is smoother than the individual contributions resulting from the higher angular momentum states.

In some representative examples it is shown that those half-shell amplitudes can differ significantly depending on the potential employed. Of course, the observable quantities, the on-shell amplitudes, are equal since they describe the same $N N$ data base. We display the magnitudes of the physical $t$-matrix elements derived from the Bonn-B model as a function of scattering angle and energy. Though the $N N$ potential is tuned only up to about $350 \mathrm{MeV}$ our results are shown up to $1 \mathrm{GeV}$ to demonstrate the ease by which also high energies can be handled in our approach without partial waves.

We would like to emphasize that the scheme developed here is algebraically very simple to handle provided potentials are given in an operator form. This is, e.g., the case for all interactions developed within a field theoretic frame work. In addition, the solution of two dimensional integral equations does not pose any difficulty for modern computers. 
This work is intended to serve as starting point towards treating three-nucleon scattering without partial waves. In Refs. [6,7] three-nucleon bound and scattering states have already been treated without partial waves, however, neglecting spin degrees of freedom. In the present work we show at the two-nucleon level that the inclusion of spin degrees of freedom is quite readily possible. Future work needs to combine the experience with the $N N$ system gained here with the three-body calculations without partial waves of Refs. [6,7]. This will be an important step forward since present $3 \mathrm{~N}$ scattering codes based on partial wave methods reach the limit of today's supercomputers already at intermediate energies be- low the pion threshold. Future applications at higher energies require new techniques.

\section{ACKNOWLEDGMENTS}

This work was performed in part under the auspices of the Deutsche Akademische Austauschdienst under Contract No. A/96/32258, the U.S. Department of Energy under Contract No. DE-FG02-93ER40756 with Ohio University, the NATO Collaborative Research Grant 960892, and the National Science Foundation under Grant No. INT-9726624. We thank the Computer Center of the RWTH Aachen for the use of their facilities under Grant No. P039.

\section{APPENDIX A: THE TRANSFORMATION MATRIX $A$}

In this appendix we explicitly give the transformation from the Wolfenstein operators given in Eqs. (3.1) to the operators $\Omega_{i}$ of Eqs. (3.6), i.e., the matrix $A_{i j}$ indicated in Eq. (3.7). The matrix is explicitly given as

$$
A=\left(\begin{array}{cccccc}
1 & 0 & 0 & 0 & 0 & 0 \\
0 & \frac{i a}{\gamma} & \frac{-2 i}{\gamma a} & \frac{2 i}{a} & \frac{2 i}{\gamma a} & \frac{-2 i}{\gamma a} \\
-1 & 1 & 0 & \frac{2 \gamma}{a^{2}} & \frac{-2}{a^{2}} & 0 \\
-1 & \frac{-q^{\prime} q a^{2}}{\gamma c} & \frac{2 q^{\prime}\left(q^{\prime} \gamma+q\right)}{\gamma c} & \frac{2 q^{\prime} q}{c} & \frac{-2 q^{\prime} q}{\gamma c} & \frac{2 q\left(q \gamma+q^{\prime}\right)}{\gamma c} \\
-1 & \frac{q^{\prime} q a^{2}}{\gamma b} & \frac{2 q^{\prime}\left(q^{\prime} \gamma-q\right)}{\gamma b} & \frac{-2 q^{\prime} q}{b} & \frac{2 q^{\prime} q}{\gamma b} & \frac{2 q\left(q \gamma-q^{\prime}\right)}{\gamma b} \\
\frac{-2\left(q^{\prime 2}-q^{2}\right)}{e} & 0 & \frac{4 q^{\prime 2}}{e} & 0 & 0 & \frac{-4 q^{2}}{e}
\end{array}\right) .
$$

The matrix elements $A_{i j}$ are scalar functions. We calculate

$$
\operatorname{det} A=\frac{-i 2^{9}}{\gamma \sqrt{1-\gamma^{2}}}\left(\frac{q q^{\prime}}{\left|\mathbf{q}^{\prime}+\mathbf{q}\right|\left|\mathbf{q}^{\prime}-\mathbf{q}\right|}\right)^{3},
$$

and find the inverse matrix $A_{i j}^{-1}$ to be

$$
A^{-1}=\left|\begin{array}{cccccc}
1 & 0 & 0 & 0 & 0 & 0 \\
\frac{3}{2} & 0 & \frac{1}{2} & \frac{e^{2}}{8 a^{2} q^{\prime 2} q^{2}} & \frac{e^{2}}{8 a^{2} q^{\prime 2} q^{2}} & \frac{-\left(q^{\prime 2}-q^{2}\right) e}{8 a^{2} q^{\prime 2} q^{2}} \\
\frac{1}{2} & 0 & 0 & \frac{c}{8 q^{\prime 2}} & \frac{b}{8 q^{\prime 2}} & \frac{e}{8 q^{\prime 2}} \\
\frac{\gamma}{2} & \frac{-i a}{4} & 0 & \frac{c}{8 q^{\prime} q} & \frac{-b}{8 q^{\prime} q} & 0 \\
\frac{1+\gamma^{2}}{4} & \frac{-i a \gamma}{4} & \frac{-a^{2}}{4} & \frac{f c}{16} & \frac{f b}{16} & \frac{g e}{16} \\
\frac{1}{2} & 0 & 0 & \frac{c}{8 q^{2}} & \frac{b}{8 q^{2}} & \frac{-e}{8 q^{2}}
\end{array}\right| .
$$


For both matrices we introduced the abbreviations

$$
\begin{aligned}
& a=\sqrt{1-\gamma^{2}}, \quad b=q^{\prime 2}+q^{2}-2 q^{\prime} q \gamma, \quad c=q^{\prime 2}+q^{2}+2 q^{\prime} q \gamma, \\
& e=\sqrt{\left(q^{\prime 2}+q^{2}\right)^{2}-4 q^{\prime 2} q^{2} \gamma^{2}}, \quad f=\frac{1}{q^{\prime 2}}+\frac{1}{q^{2}}, \quad g=\frac{1}{q^{\prime 2}}-\frac{1}{q^{2}} .
\end{aligned}
$$

APPENDIX B: THE OBE POTENTIAL AS FUNCTION OF WOLFENSTEIN OPERATORS

In terms of the Wolfenstein operators $W_{i}$ of Eq. (3.1), the Bonn-B potential takes the following form:

$$
\begin{gathered}
V_{p s}\left(\mathbf{q}^{\prime}, \mathbf{q}\right)=\frac{g_{p s}^{2}}{(2 \pi)^{3} 4 m^{2}} \sqrt{\frac{m}{E^{\prime}}} \sqrt{\frac{m}{E} \frac{F_{p s}^{2}\left[\left(\mathbf{q}^{\prime}-\mathbf{q}\right)^{2}\right]}{\left(\mathbf{q}^{\prime}-\mathbf{q}\right)^{2}+m_{p s}^{2}} \frac{\hat{O}_{p s}}{W^{\prime} W},} \\
V_{s}\left(\mathbf{q}^{\prime}, \mathbf{q}\right)=\frac{g_{s}^{2}}{(2 \pi)^{3} 4 m^{2}} \sqrt{\frac{m}{E^{\prime}}} \sqrt{\frac{m}{E} \frac{F_{s}^{2}\left[\left(\mathbf{q}^{\prime}-\mathbf{q}\right)^{2}\right]}{\left(\mathbf{q}^{\prime}-\mathbf{q}\right)^{2}+m_{s}^{2}} \frac{\hat{O}_{s}}{W^{\prime} W}}, \\
V_{v}\left(\mathbf{q}^{\prime}, \mathbf{q}\right)=\frac{1}{(2 \pi)^{3} 4 m^{2}} \sqrt{\frac{m}{E^{\prime}}} \sqrt{\frac{m}{E}} \frac{F_{v}^{2}\left[\left(\mathbf{q}^{\prime}-\mathbf{q}\right)^{2}\right]}{\left(\mathbf{q}^{\prime}-\mathbf{q}\right)^{2}+m_{v}^{2}} \frac{\left(g_{v}^{2} \hat{O}_{v v}+2 g_{v} f_{v} \hat{O}_{v t}+f_{v}^{2} \hat{O}_{t t}\right)}{W^{\prime} W},
\end{gathered}
$$

where $m$ denotes the nucleon mass and $W=\sqrt{m^{2}+\mathbf{q}^{2}}$. The operators $\hat{O}_{p s, s, v}$ are given as

$$
\begin{aligned}
& \hat{O}_{p s}=-\frac{1}{4}\left[\left(W^{\prime}-W\right)^{2}\left(q^{\prime 2}+q^{2}+2 q^{\prime} q \gamma\right) \mathbf{W}_{\mathbf{4}}+\left(W^{\prime}+W\right)^{2}\left(q^{\prime 2}+q^{2}-2 q^{\prime} q \gamma\right) \mathbf{W}_{\mathbf{5}}\right]-\left(W^{\prime 2}-W^{2}\right) \sqrt{\left(q^{\prime 2}+q^{2}\right)^{2}-4 q^{\prime 2} q^{2} \gamma^{2} \mathbf{W}_{\mathbf{6}},} \\
& \hat{O}_{s}=-\left(W^{\prime} W-q^{\prime} q \gamma\right)^{2} \mathbf{W}_{\mathbf{1}}-i\left(W^{\prime} W-q^{\prime} q \gamma\right) q^{\prime} q \sqrt{1-\gamma^{2}} \mathbf{W}_{\mathbf{2}}+q^{\prime 2} q^{2}\left(1-\gamma^{2}\right) \mathbf{W}_{\mathbf{3}} \\
& \hat{O}_{v v}=\left(W^{\prime 2} W^{2}+q^{\prime 2} q^{2} \gamma^{2}+4 W^{\prime} W q^{\prime} q \gamma+W^{\prime 2} q^{2}+W^{2} q^{\prime 2}\right) \mathbf{W}_{\mathbf{1}}-i\left(3 W^{\prime} W+q^{\prime} q \gamma\right) q^{\prime} q \sqrt{1-\gamma^{2}} \mathbf{W}_{\mathbf{2}} \\
&-\left[1-\gamma^{2}+\left(W^{\prime 2} q^{2}+W^{2} q^{\prime 2}-2 W^{\prime} W q^{\prime} q \gamma\right)\right] \mathbf{W}_{\mathbf{3}}+\left[\left(W^{\prime 2}+W^{2}-2 W^{\prime} W\right)\right. \\
&\left.-\frac{\left(q^{\prime 2}+q^{2}-2 q^{\prime} q \gamma\right)}{q^{\prime 2} q^{2}\left(1-\gamma^{2}\right)}\left(W^{\prime 2} q^{2}+W^{2} q^{\prime 2}-2 W^{\prime} W q^{\prime} q \gamma\right)\right] \frac{1}{4}\left(q^{\prime 2}+q^{2}+2 q^{\prime} q \gamma\right) \mathbf{W}_{\mathbf{4}}+\left[\left(W^{\prime 2}+W^{2}+2 W^{\prime} W\right)\right. \\
&\left.-\frac{\left(q^{\prime 2}+q^{2}+2 q^{\prime} q \gamma\right)}{q^{\prime 2} q^{2}\left(1-\gamma^{2}\right)}\left(W^{\prime 2} q^{2}+W^{2} q^{\prime 2}-2 W^{\prime} W q^{\prime} q \gamma\right)\right] \frac{1}{4}\left(q^{\prime 2}+q^{2}-2 q^{\prime} q \gamma\right) \mathbf{W}_{\mathbf{5}}-\left[\left(W^{\prime 2}-W^{2}\right)\right. \\
&\left.-\frac{\left(q^{\prime 2}-q^{2}\right)}{q^{\prime 2} q^{2}\left(1-\gamma^{2}\right)}\left(W^{\prime 2} q^{2}+W^{2} q^{\prime 2}-2 W^{\prime} W q^{\prime} q \gamma\right)\right] \frac{1}{4} \sqrt{\left(q^{\prime 2}+q^{2}\right)^{2}-4 q^{\prime 2} q^{2} \gamma^{2} \mathbf{W}_{\mathbf{6}},}
\end{aligned}
$$




$$
\begin{aligned}
\hat{O}_{v t}= & {\left[\frac{\left(2 m-E^{\prime}-E\right)}{m} W^{\prime 2} W^{2}+\frac{\left(W^{\prime}+W\right)}{m} q^{\prime 2} q^{2} \gamma^{2}+2 q^{\prime} q W^{\prime} W \gamma \frac{\left(2 m-E^{\prime}+E\right)}{2 m} W^{\prime 2} q^{2}+\frac{\left(2 m+E^{\prime}-E\right)}{2 m} W^{2} q^{\prime 2}\right] \mathbf{W}_{\mathbf{1}} } \\
& -i\left[\frac{\left(W^{\prime}+W\right)}{m} q^{\prime} q \gamma+2 W^{\prime} W\right] q^{\prime} q \sqrt{1-\gamma^{2}} \mathbf{W}_{\mathbf{2}}-\left\{\frac{\left(W^{\prime}+W\right)}{m}\left(1-\gamma^{2}\right)+\left[\frac{\left(2 m-E^{\prime}+E\right)}{2 m} W^{\prime 2} q^{2}+\frac{\left(2 m+E^{\prime}-E\right)}{2 m} W^{2} q^{\prime 2}\right.\right. \\
& \left.\left.-2 W^{\prime} W q^{\prime} q \gamma\right]\right\} \mathbf{W}_{\mathbf{3}}+\left\{\frac{\left(2 m-E^{\prime}+E\right)}{2 m} W^{\prime 2}+\frac{\left(2 m+E^{\prime}-E\right)}{2 m} W^{2}-2 W^{\prime} W-\left[\frac{\left(2 m-E^{\prime}+E\right)}{2 m} \frac{W^{\prime 2}}{q^{\prime 2}}+\frac{\left(2 m+E^{\prime}-E\right)}{2 m} \frac{W^{2}}{q^{2}}\right.\right. \\
& \left.\left.-2 W^{\prime} W \frac{\gamma}{q^{\prime} q}\right] \times \frac{\left(q^{\prime 2}+q^{2}-2 q^{\prime} q \gamma\right)}{\left(1-\gamma^{2}\right)}\right\} \frac{1}{4}\left(q^{\prime 2}+q^{2}+2 q^{\prime} q \gamma\right) \mathbf{W}_{\mathbf{4}}+\left\{\frac{\left(2 m-E^{\prime}+E\right)}{2 m} W^{\prime 2}+\frac{\left(2 m+E^{\prime}-E\right)}{2 m} W^{2}+2 W^{\prime} W\right. \\
& \left.-\left[\frac{\left(2 m-E^{\prime}+E\right)}{2 m} \frac{W^{\prime 2}}{q^{\prime 2}}+\frac{\left(2 m+E^{\prime}-E\right)}{2 m} \frac{W^{2}}{q^{2}}-2 W^{\prime} W \frac{\gamma}{q^{\prime} q}\right] \frac{\left(q^{\prime 2}+q^{2}+2 q^{\prime} q \gamma\right)}{\left(1-\gamma^{2}\right)}\right\} \frac{1}{4}\left(q^{\prime 2}+q^{2}-2 q^{\prime} q \gamma\right) \mathbf{W}_{\mathbf{5}} \\
& -\left\{\frac{\left(2 m-E^{\prime}+E\right)}{2 m} W^{\prime 2}-\frac{\left(2 m+E^{\prime}-E\right)}{2 m} W-\left[\frac{\left(2 m-E^{\prime}+E\right)}{2 m} \frac{W^{\prime 2}}{q^{\prime 2}}+\frac{\left(2 m+E^{\prime}-E\right)}{2 m} \frac{W^{2}}{q^{2}}\right.\right. \\
& \left.-2 W^{\prime} W \frac{\gamma}{q^{\prime} q}\right]\left(\frac{\left(q^{\prime 2}-q^{2}\right)}{\left(1-\gamma^{2}\right)}\right\} \frac{1}{4} \sqrt{\left(q^{\prime 2}+q^{2}\right)^{2}-4 q^{\prime 2} q^{2} \gamma^{2}} \mathbf{W}_{\mathbf{6}}
\end{aligned}
$$$$
\hat{O}_{t t}=\left\{\left[5 m^{2}-4 m\left(E^{\prime}+E\right)+3 E^{\prime} E+q^{\prime} q \gamma\right] W^{\prime 2} W+\left[5 m^{2}+4 m\left(E^{\prime}+E\right)+3 E^{\prime} E+q^{\prime} q \gamma\right] q^{\prime 2} q^{2} \gamma^{2}+\left(2 m^{2}-4 E^{\prime} E-E^{\prime 2}-E^{2}\right.\right.
$$$$
\left.\left.-2 q^{\prime} q \gamma\right) W^{\prime} W q^{\prime} q \gamma+\frac{\left(2 m-E^{\prime}+E\right)^{2}}{2} q^{2} W^{\prime 2}+\frac{\left(2 m+E^{\prime}-E\right)^{2}}{2} q^{\prime 2} W^{2}\right\} \frac{1}{2 m^{2}} \mathbf{W}_{\mathbf{1}}-i\left\{\left(3 m^{2}-E^{\prime} E-E^{\prime 2}-E^{2}\right.\right.
$$$$
\left.\left.-q^{\prime} q \gamma\right) W^{\prime} W+\left[5 m^{2}+4 m\left(E^{\prime}+E\right)+3 E^{\prime} E+q^{\prime} q \gamma\right] q^{\prime} q \gamma\right\} \frac{q^{\prime} q}{2 m^{2}} \sqrt{1-\gamma^{2}} \quad \mathbf{W}_{2}-\left\{\left[10 m^{2}+8 m\left(E^{\prime}+E\right)+6 E^{\prime} E+2 q^{\prime} q \gamma\right]\right.
$$$$
\left.\times\left(1-\gamma^{2}\right)+\left[\left(2 m-E^{\prime}+E\right)^{2} W^{\prime 2} q^{2}+\left(2 m+E^{\prime}-E\right)^{2} W^{2} q^{\prime 2}-2\left[4 m^{2}-\left(E^{\prime}-E\right)^{2}\right] W^{\prime} W q^{\prime} q \gamma\right]\right\} \frac{W_{3}}{4 m^{2}}
$$$$
+\left\{\left(2 m-E^{\prime}+E\right)^{2} W^{\prime 2}+\left(2 m+E^{\prime}-E\right)^{2} W^{2}-2\left[4 m^{2}-\left(E^{\prime}-E\right)^{2}\right] W^{\prime} W-\left[\left(2 m-E^{\prime}+E\right)^{2} \frac{W^{\prime 2}}{q^{\prime 2}}+\left(2 m+E^{\prime}-E\right)^{2} \frac{W^{2}}{q^{2}}\right.\right.
$$$$
\left.\left.-2\left[4 m^{2}-\left(E^{\prime}-E\right)^{2}\right] W^{\prime} W \frac{\gamma}{q^{\prime} q}\right] \frac{\left(q^{\prime 2}+q^{2}-2 q^{\prime} q \gamma\right)}{\left(1-\gamma^{2}\right)}\right\} \frac{\left(q^{\prime 2}+q^{2}+2 q^{\prime} q \gamma\right)}{16 m^{2}} \mathbf{W}_{\mathbf{4}}+\left\{\left(2 m-E^{\prime}+E\right)^{2} W^{\prime 2}\right.
$$$$
+\left(2 m+E^{\prime}-E\right)^{2} W^{2}+2\left[4 m^{2}-\left(E^{\prime}-E\right)^{2}\right] W^{\prime} W-\left[\left(2 m-E^{\prime}+E\right)^{2} \frac{W^{\prime 2}}{q^{\prime 2}}+\left(2 m+E^{\prime}-E\right)^{2} \frac{W^{2}}{q^{2}}\right.
$$$$
\left.\left.-2\left[4 m^{2}-\left(E^{\prime}-E\right)^{2}\right] W^{\prime} W \frac{\gamma}{q^{\prime} q}\right]\left(q^{\prime 2}+q^{2}+2 q^{\prime} q \gamma\right) \frac{1}{\left(1-\gamma^{2}\right)}\right\} \frac{\left(q^{\prime 2}+q^{2}-2 q^{\prime} q \gamma\right)}{16 m^{2}} \mathbf{W}_{5}-\left\{\left(2 m-E^{\prime}+E\right)^{2} W^{\prime 2}\right.
$$$$
-\left(2 m+E^{\prime}-E\right)^{2} W^{2}-\left[\left(2 m-E^{\prime}+E\right)^{2} \frac{W^{\prime 2}}{q^{\prime 2}}+\left(2 m+E^{\prime}-E\right)^{2} \frac{W^{2}}{q^{2}}-2\left[4 m^{2}-\left(E^{\prime}-E\right)^{2}\right] W^{\prime} W \frac{\gamma}{q^{\prime} q}\right]
$$$$
\left.\times \frac{\left(q^{\prime 2}-q^{2}\right)}{\left(1-\gamma^{2}\right)}\right\} \frac{\sqrt{\left(q^{\prime 2}+q^{2}\right)^{2}-4 q^{\prime 2} q^{2} \gamma^{2}}}{16 m^{2}} \mathbf{W}_{\mathbf{6}} .
$$ 


\section{APPENDIX C: THE ARGONNE AV18 POTENTIAL AS FUNCTION OF WOLFENSTEIN OPERATORS}

In terms of the Wolfenstein operators $W_{i}$ of Eq. (3.1), the AV18 potential takes the following form:

$$
\begin{aligned}
& v_{S T}^{c}\left(\mathbf{q}^{\prime}, \mathbf{q}\right)=\mathbf{W}_{\mathbf{1}} \frac{1}{2 \pi^{2}} \int_{0}^{\infty} d r r^{2} j_{0}(\rho r) v_{S T}^{c}(r), \\
& v_{S T}^{t}\left(\mathbf{q}^{\prime}, \mathbf{q}\right)=\left[\mathbf{W}_{\mathbf{3}}-\frac{1}{4} \frac{\left(\mathbf{q}^{\prime}-\mathbf{q}\right)^{2}\left(\mathbf{q}^{\prime}+\mathbf{q}\right)^{2}}{q^{\prime 2} q^{2}\left(\gamma^{2}-1\right)} \mathbf{W}_{\mathbf{4}}-\frac{1}{4} \frac{\left(q^{\prime 4}+8 q^{\prime 2} q^{2} \gamma^{2}-10 q^{\prime 2} q^{2}+4 q^{2}\right)}{q^{\prime 2} q^{2}\left(\gamma^{2}-1\right)} \mathbf{W}_{\mathbf{5}}\right. \\
& \left.+\frac{1}{4} \frac{\sqrt{\left(q^{\prime 2}+q^{2}\right)^{2}-4 q^{\prime 2} q^{2} \gamma^{2}}\left(q^{\prime 2}-q^{2}\right)}{q^{\prime 2} q^{2}\left(\gamma^{2}-1\right)} \mathbf{W}_{\mathbf{6}}\right] \frac{1}{2 \pi^{2}} \frac{1}{\rho^{2}} \int_{0}^{\infty} d r r^{2} j_{2}(\rho r) v_{S T}^{t}(r), \\
& v_{S T}^{l s}\left(\mathbf{q}^{\prime}, \mathbf{q}\right)=\frac{i}{2} q^{\prime} q \sqrt{1-\gamma^{2}} \mathbf{W}_{2} \frac{1}{2 \pi^{2}} \frac{1}{\rho} \int_{0}^{\infty} d r r^{3} j_{1}(\rho r) v_{S T}^{l s}(r), \\
& v_{S T}^{l 2}\left(\mathbf{q}^{\prime}, \mathbf{q}\right)=q^{\prime} q \gamma \mathbf{W}_{1} \frac{1}{2 \pi^{2}} \frac{2}{\rho} \int_{0}^{\infty} d r r^{3} j_{1}(\rho r) v_{S T}^{l 2}(r)-\left[q^{\prime 2} q^{2}\left(1-\gamma^{2}\right)\right] \mathbf{W}_{1} \frac{1}{2 \pi^{2}} \frac{1}{\rho^{2}} \int_{0}^{\infty} d r r^{4} j_{2}(\rho r) v_{S T}^{l 2}(r), \\
& v_{S T}^{l s 2}\left(\mathbf{q}^{\prime}, \mathbf{q}\right)=\left[q^{\prime} q \gamma \mathbf{W}_{\mathbf{1}}-\frac{i}{4} q^{\prime} q \sqrt{1-\gamma^{2}} \mathbf{W}_{\mathbf{2}}+\frac{1}{2} q^{\prime} q \gamma \mathbf{W}_{\mathbf{3}}-\frac{1}{8} \frac{\left(\mathbf{q}^{\prime}+\mathbf{q}\right)^{2}\left(q^{\prime}-\gamma q\right)\left(q^{\prime} \gamma-q\right)}{q^{\prime} q\left(\gamma^{2}-1\right)} \mathbf{W}_{\mathbf{4}}\right. \\
& \left.-\frac{1}{8} \frac{\left(\mathbf{q}^{\prime}-\mathbf{q}\right)^{2}\left(q^{\prime}+q \gamma\right)\left(q+q^{\prime} \gamma\right)}{q^{\prime} q\left(\gamma^{2}-1\right)} \mathbf{W}_{5}+\frac{1}{8} \frac{\gamma\left(q^{\prime 2}-q^{2}\right) \sqrt{\left(q^{\prime 2}+q^{2}\right)^{2}-4 q^{\prime 2} q^{2} \gamma^{2}}}{q^{\prime} q\left(\gamma^{2}-1\right)} \mathbf{W}_{\mathbf{6}}\right] \\
& \times \frac{1}{2 \pi^{2}} \frac{1}{\rho} \int_{0}^{\infty} d r r^{3} j_{1}(\rho r) v_{S T}^{l s 2}(r)+\frac{1}{2} q^{\prime 2} q^{2}\left(\gamma^{2}-1\right)\left(\mathbf{W}_{\mathbf{1}}+\mathbf{W}_{\mathbf{3}}\right) \frac{1}{2 \pi^{2}} \frac{1}{\rho^{2}} \int_{0}^{\infty} d r r^{4} j_{2}(\rho r) v_{S T}^{l s 2}(r)
\end{aligned}
$$

In our actual calculation the potential enters described by the set of operators $\Omega_{i}$ of Eq. (3.6). In order to display the relative simple form the potential takes in these operators, we also want to explicitly show them:

$$
\begin{aligned}
& v_{S T}^{c}\left(\mathbf{q}^{\prime}, \mathbf{q}\right)=\mathbf{\Omega}_{1} \frac{1}{2 \pi^{2}} \int_{0}^{\infty} d r r^{2} j_{0}(\rho r) v_{S T}^{c}(r), \\
& v_{S T}^{t}\left(\mathbf{q}^{\prime}, \mathbf{q}\right)=\left[6 q^{\prime} q \mathbf{\Omega}_{\mathbf{4}}+2 q^{\prime 2}\left(\mathbf{\Omega}_{\mathbf{2}}-3 \mathbf{\Omega}_{\mathbf{3}}\right)+2 q^{2}\left(\mathbf{\Omega}_{\mathbf{2}}-3 \mathbf{\Omega}_{\mathbf{6}}\right)-q^{\prime} q \gamma \mathbf{\Omega}_{\mathbf{2}}-3 q^{\prime} q \frac{1}{\gamma}\right. \\
& \left.\times\left(\boldsymbol{\Omega}_{\mathbf{2}}-2 \boldsymbol{\Omega}_{\mathbf{3}}-2 \boldsymbol{\Omega}_{\mathbf{6}}+2 \boldsymbol{\Omega}_{\mathbf{5}}\right)\right] \frac{1}{2 \pi^{2}} \frac{1}{\rho^{2}} \int_{0}^{\infty} d r r^{2} j_{2}(\rho r) v_{S T}^{t}(r), \\
& v_{S T}^{l s}\left(\mathbf{q}^{\prime}, \mathbf{q}\right)=-\frac{1}{2} q^{\prime} q\left[2 \mathbf{\Omega}_{\mathbf{4}}-\gamma \mathbf{\Omega}_{\mathbf{2}}+\frac{1}{\gamma}\left(\mathbf{\Omega}_{\mathbf{2}}-2 \mathbf{\Omega}_{\mathbf{3}}-2 \mathbf{\Omega}_{\mathbf{6}}+2 \mathbf{\Omega}_{\mathbf{5}}\right)\right] \frac{1}{2 \pi^{2}} \frac{1}{\rho} \int_{0}^{\infty} d r r^{3} j_{1}(\rho r) v_{S T}^{l s}(r), \\
& v_{S T}^{l 2}\left(\mathbf{q}^{\prime}, \mathbf{q}\right)=q^{\prime} q \gamma \mathbf{\Omega}_{1} \frac{1}{2 \pi^{2}} \frac{2}{\rho} \int_{0}^{\infty} d r r^{3} j_{1}(\rho r) v_{S T}^{l 2}(r)-\mathbf{\Omega}_{1}\left[q^{\prime 2} q^{2}\left(1-\gamma^{2}\right)\right] \frac{1}{2 \pi^{2}} \frac{1}{\rho^{2}} \int_{0}^{\infty} d r r^{4} j_{2}(\rho r) v_{S T}^{l 2}(r), \\
& v_{S T}^{l s 2}\left(\mathbf{q}^{\prime}, \mathbf{q}\right)=\frac{1}{2} q^{\prime} q\left(\gamma \mathbf{\Omega}_{\mathbf{2}}+\frac{1}{\gamma}\left(\mathbf{\Omega}_{\mathbf{2}}+2 \mathbf{\Omega}_{\mathbf{5}}-2 \mathbf{\Omega}_{\mathbf{3}}-2 \mathbf{\Omega}_{\mathbf{6}}\right)\right) \frac{1}{2 \pi^{2}} \frac{1}{\rho} \int_{0}^{\infty} d r r^{3} j_{1}(\rho r) v_{S T}^{l s 2}(r) \\
& -\frac{1}{2} q^{\prime 2} q^{2}\left[\left(1-\gamma^{2}\right) \mathbf{\Omega}_{\mathbf{2}}+2 \gamma \mathbf{\Omega}_{\mathbf{4}}-2 \boldsymbol{\Omega}_{\mathbf{5}}\right] \frac{1}{2 \pi^{2}} \frac{1}{\rho^{2}} \int_{0}^{\infty} d r r^{4} j_{2}(\rho r) v_{S T}^{l s 2}(r) .
\end{aligned}
$$


[1] W. Glöckle, H. Witala, D. Hüber, H. Kamada, and J. Golak, Phys. Rep. 274, 107 (1996).

[2] A. Nogga, D. Hüber, H. Kamada, and W. Glöckle, Phys. Lett. B 409, 19 (1997).

[3] W. Glöckle and H. Kamada, Phys. Rev. Lett. 71, 971 (1993).

[4] W. Glöckle, The Quantum Mechanical Few-Body Problem (Springer-Verlag, Berlin, 1983).

[5] D. Hüber, H. Witala, A. Nogga, W. Glöckle, and H. Kamada, Few-Body Syst. 22, 107 (1997).

[6] Ch. Elster, W. Schadow, A. Nogga, and W. Glöckle, FewBody Syst. 27, 83 (1999).

[7] W. Schadow, Ch. Elster, and W. Glöckle, Few-Body Syst. 28, 15 (2000).

[8] Ch. Elster, J. H. Thomas, and W. Glöckle, Few-Body Syst. 24, 55 (1998).

[9] A. Delfino and E. F. Redish, XIII International Conference on Few-Body Problems in Physics, Adelaide, 1992, Book of Contributions, edited by I. R. Afnan and R. T. Cahill, p. 148.

[10] H. Witala, H. Kamada, A. Nogga, W. Glöckle, Ch. Elster, and
D. Hüber, Phys. Rev. C 59, 3035 (1999).

[11] J. Carlson and R. Schiavilla, Rev. Mod. Phys. 70, 743 (1998).

[12] J. Holz and W. Glöckle, Phys. Rev. C 37, 1386 (1988).

[13] B. terHaar and R. Malfliet, Phys. Rep. 149, 207 (1987).

[14] R. A. Rice and Y. E. Kim, Few-Body Syst. 14, 127 (1993).

[15] R. Machleidt, Adv. Nucl. Phys. 19, 189 (1989).

[16] R. B. Wiringa, V. G. J. Stoks, and R. Schiavilla, Phys. Rev. C 51, 38 (1995).

[17] V. G. J. Stoks, R. A. M. Klomp, C. P. F. Terheggen, and J. J. de Swart, Phys. Rev. C 49, 2950 (1994).

[18] R. Machleidt, F. Sammerruca, and Y. Song, Phys. Rev. C 53, R1483 (1996).

[19] R. Machleidt, K. Holinde, and Ch. Elster, Phys. Rep. 149, 1 (1987).

[20] L. Wolfenstein, Phys. Rev. 96, 1654 (1954).

[21] M. Abramowitz and I. A. Stegun, Handbook of Mathematical Functions (Dover, New York, 1972), p. 890.

[22] B. Wiringa (private communication). 